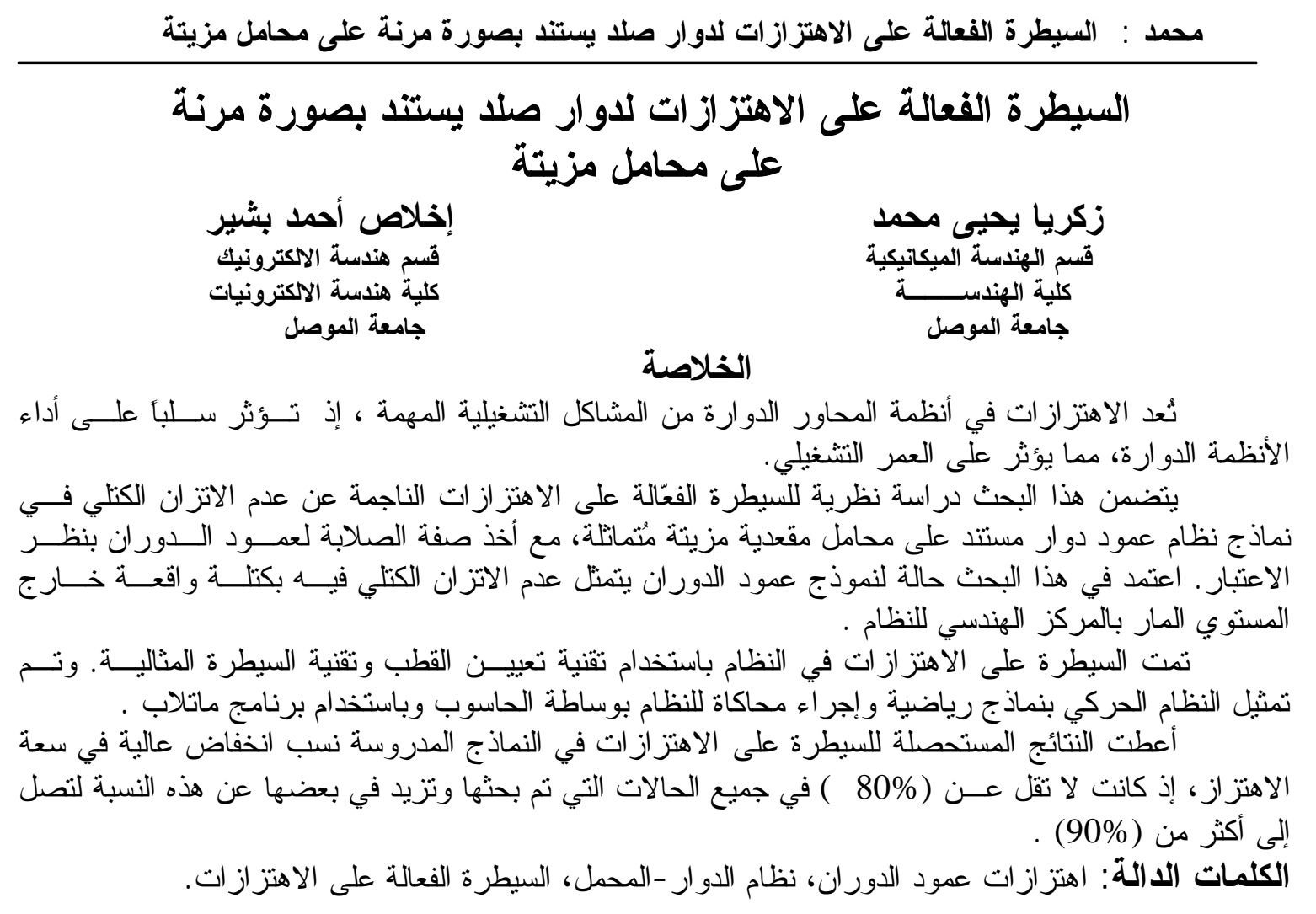

\title{
Active Control Of Vibration For Aflixibly Supported Rigid Rotor On Oil Film Bearings
}

\author{
Zakariya Y. Mohammad \\ Mechanical Engineering Department \\ College of Engineering \\ University of Mosul
}

\author{
Ikhlas A. Basheer \\ Electronics Engineering Department \\ College of Electronics Engineering \\ University of Mosul
}

\begin{abstract}
Vibrations in rotating system are considered as one of the major operating problems since they have adverse effects on the performance of rotating system, which result in operating life reduction.

In this research, the active control of vibration, as a response to mass unbalance, in a rigid rotor symmetrically supported by an oil film bearing is considered.

A model of rigid rotor is considered in this study, in which the unbalance mass is positioned out of the plane of the geometrical center of the system.

For the cases studied, the active control of system vibration was achieved by the Application of two techniques, namely, pole placement and optimal control techniques, the system dynamics is represented by mathematical models and the system is simulated by a digital computer using Matlab programs.

The results obtained showed a high reduction in vibration amplitude, This reduction was more than $80 \%$ and in some situations it raises to more than $90 \%$.
\end{abstract}

Key words: Rotor vibration, rotor bearing system, Active vibration control. 


\section{المقدمة وعرض البحوث السابقة}

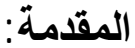

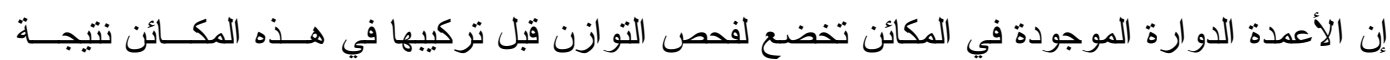

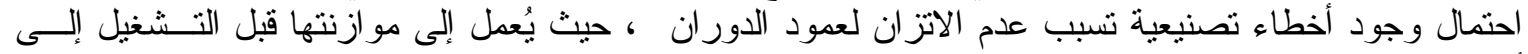

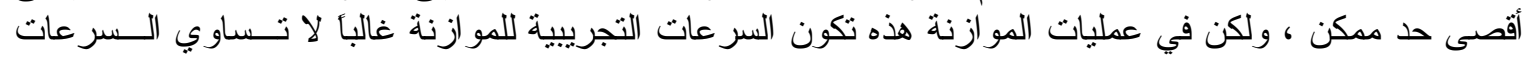

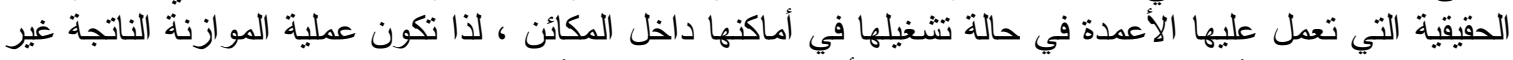

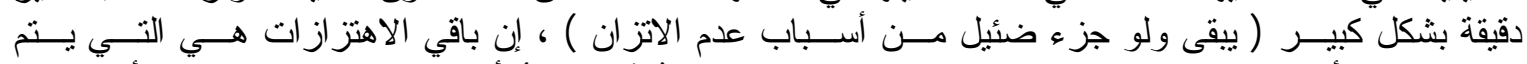

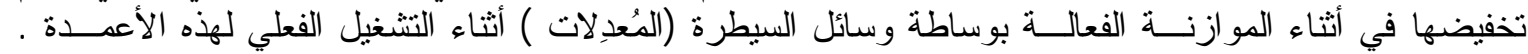

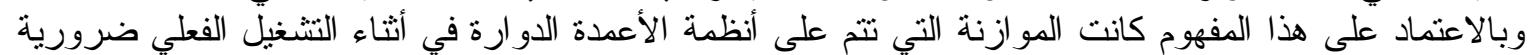

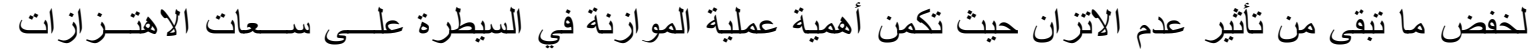

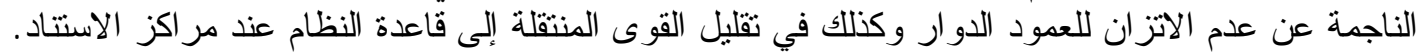

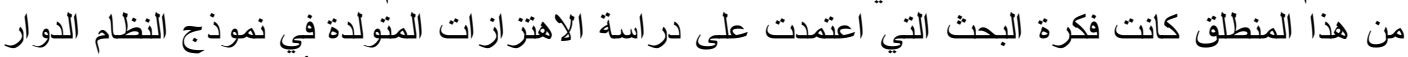

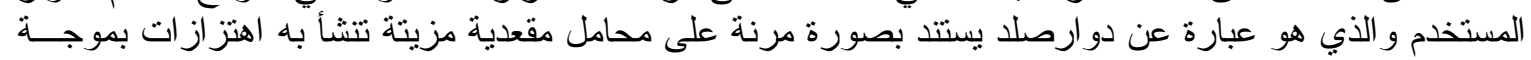

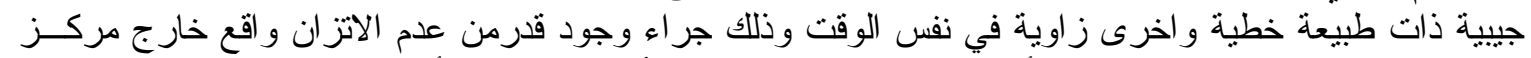

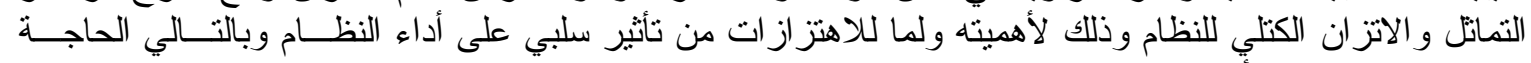

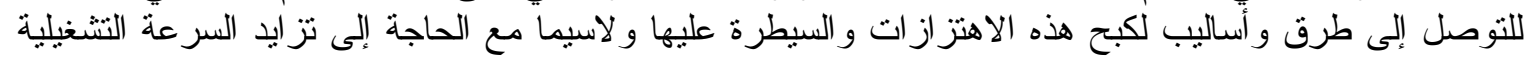

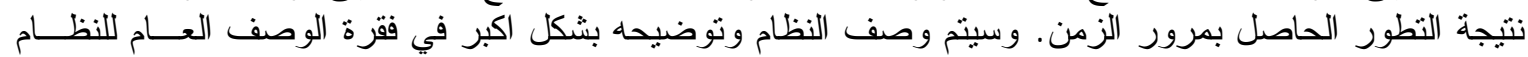

لاحقاً.

إن الدر اسات و البحوث التي تتاولت در اسة اهتزاز ات الدحاور الدوارة ومسبباتها وكيفية السيطرة عليهــا

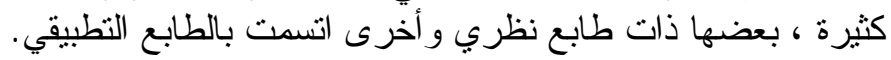

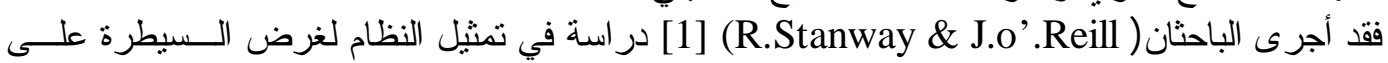

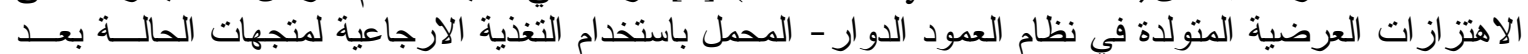

تحديد قيم آيكن في نظام الدارة المغلقة لضئة لضمان الاستقرارية.

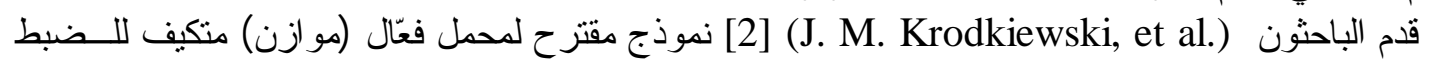

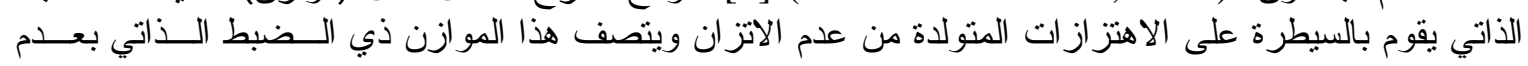

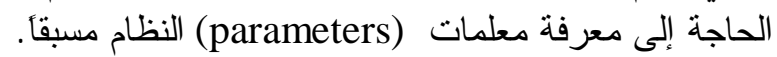

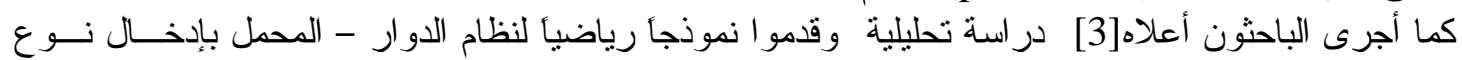

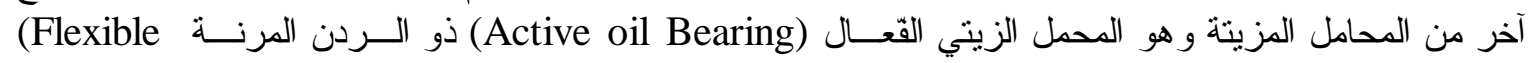
sleeve) هيدروليكية مسيطرّ عليها.

قدم الباحثان (Shiyu Zhou \& Jianjun Shi) سلسلة من البحوث و الدراسات في مجال الموازنة الفعَّالـــة

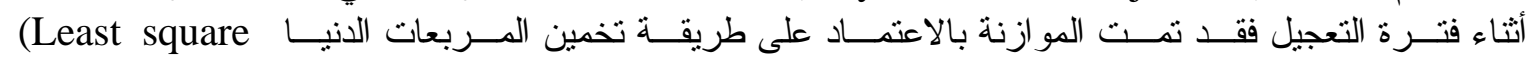
estimation)

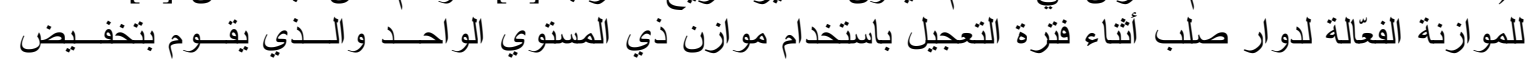

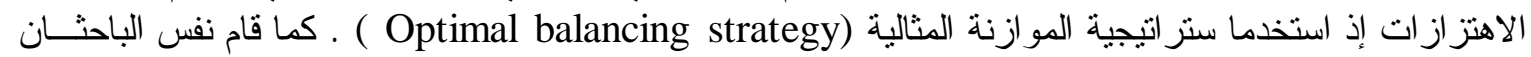

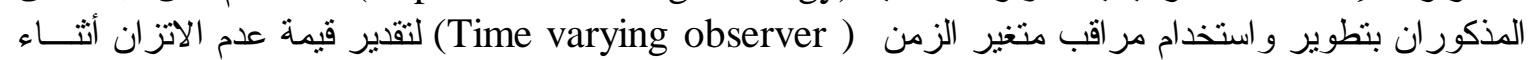

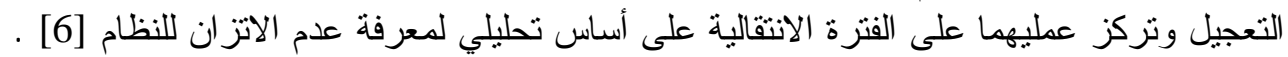

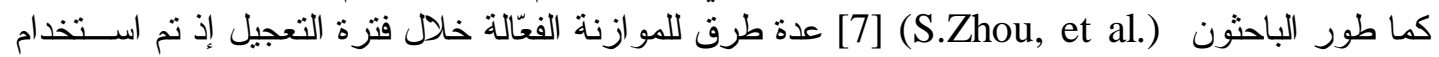

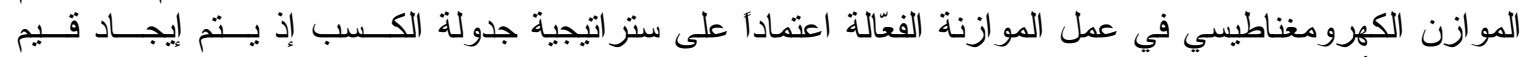

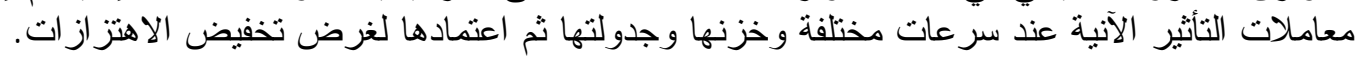

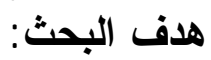

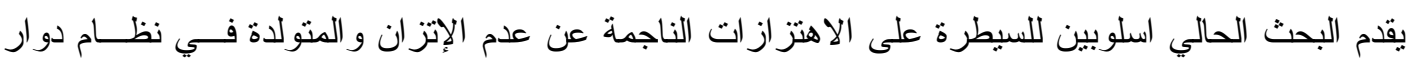

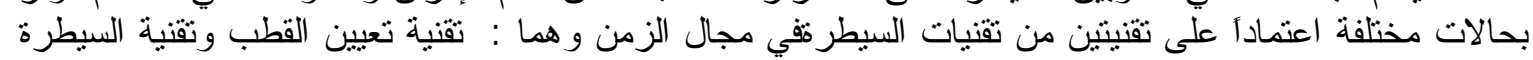

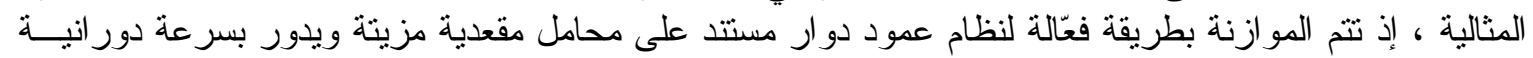

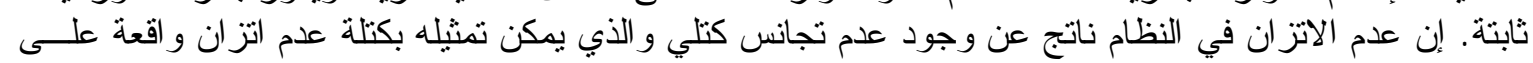
الدو ار . 


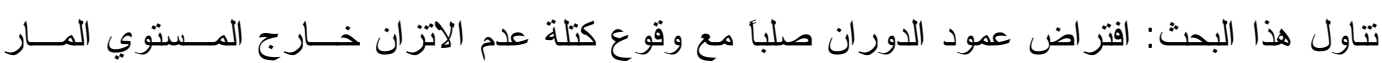

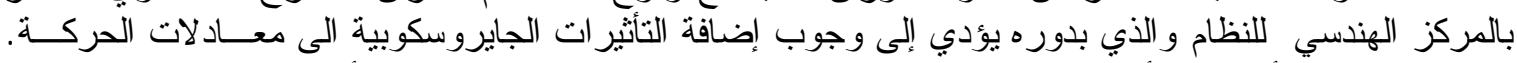

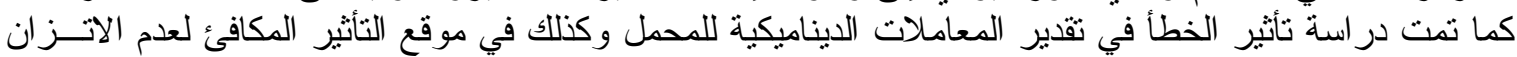

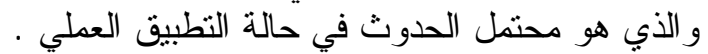

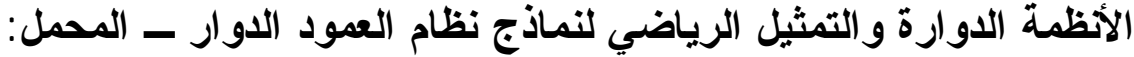

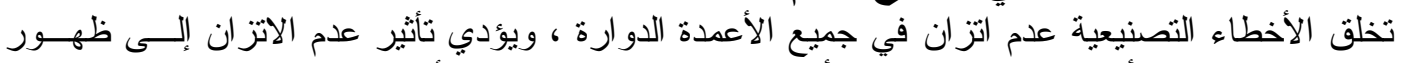

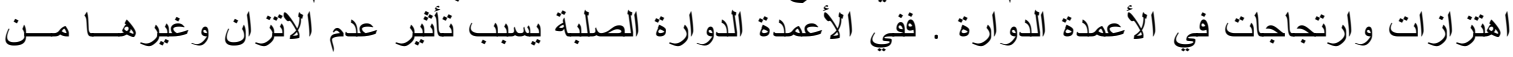

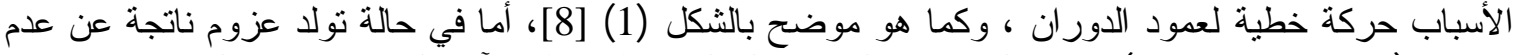

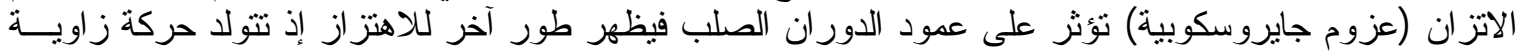

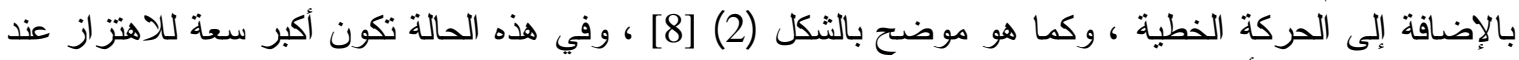

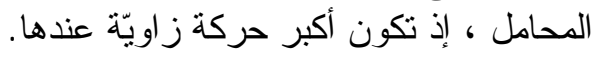

\section{وصف عام لنظام العمود الاوار - المحمل والبيانات المعتمدة للنموذج:}

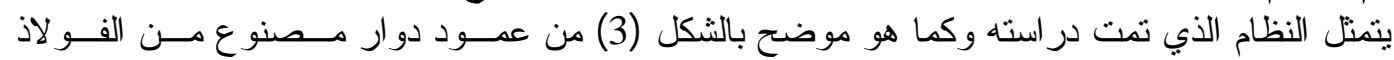

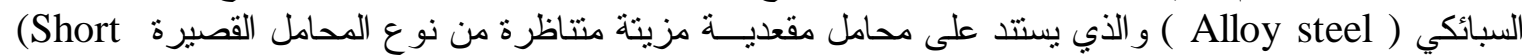
من oil film bearings)

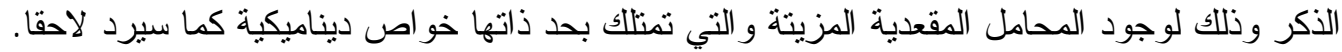

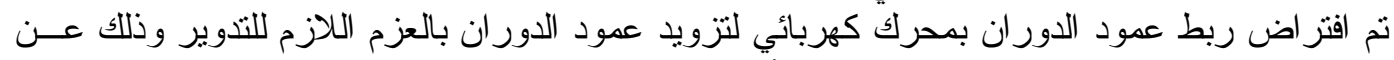

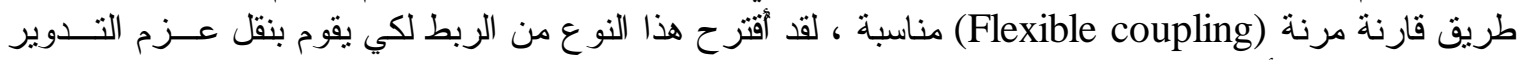

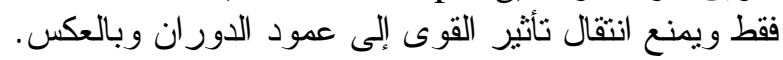

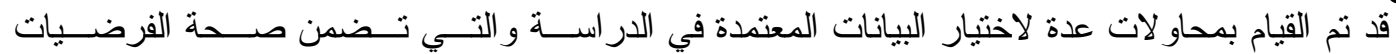

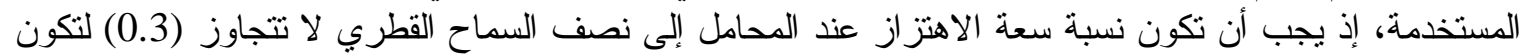

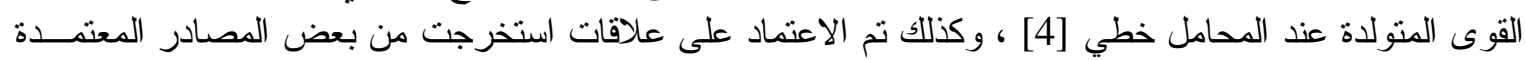

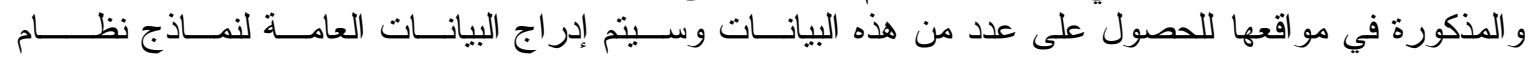
العهــود الدو ار ــ المحمل و التي تمت در استها في هذا البحث من خلال الجدول الموضح و على النحو الآتي:

الجدول (1): البيانات العامة لنماذج نظام عمود الدوران - الدحمل الني تحت الدراسة [11]

\begin{tabular}{|c|c|c|}
\hline الوحدة الوح & بيانات الاوار الصلب & الاسم و الرمز \\
\hline$m$ & 0.8 & طول العمود L \\
\hline$m$ & 0.05 & نصف قطر العمود r \\
\hline$K g \cdot m^{2}$ & 2.835 & It عزم القصور الذاتي القطري \\
\hline$K g \cdot m^{2}$ & 0.35499 & عزم القصور الذاتي القطبي \\
\hline$N / m$ & $7.8919 \times 10^{6}$ & مكافئ نابضية المحمل بالاتجاه \\
\hline$N / m$ & $-6.9483 \times 10^{5}$ & 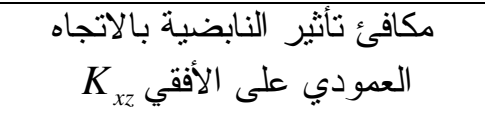 \\
\hline$N / m$ & $-1.817 \times 10^{7}$ & مكافئ تأثير النابضية بالاتجاه الأفقي \\
\hline$N / m$ & $1.222 \times 10^{7}$ & مكافئ نابضية المحمل بالاتجاه \\
\hline$N / m$ & $\infty$ & مكافئ نابضية العمود \\
\hline
\end{tabular}




\begin{tabular}{|c|c|c|}
\hline Al-Rafidain Engineering & Vol.17 & No.5 \\
\hline$N \cdot s / m$ & 31069 & مكافئ إخماد المحمل بالاتجاه \\
\hline$N \cdot s / m$ & -38775 & 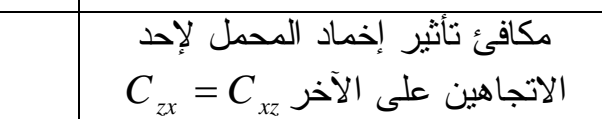 \\
\hline$N \cdot s / m$ & $1.2311 \times 10^{5}$ & 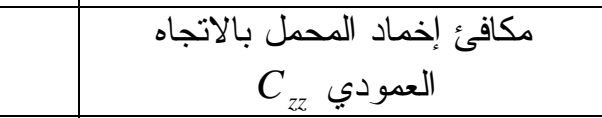 \\
\hline$m$ & $1 \times 10^{-4}$ & نصف السماح القطري c \\
\hline$K g$ & 81.691 & الكتلة الكلية للعمود M \\
\hline$K g$ & 0.01 & كتلة عدم الاتزان \\
\hline$m$ & 0.1 & عرض القرص الوسطي l \\
\hline$m$ & 0.125 & نصف قطر القرص الوسطي $R_{1}$ \\
\hline$m$ & 0.05 & البعد الأفقي لكتلة عدم الاتزان \\
\hline$m$ & 0.05 & البعد العمودي لكتلة عدم الاتزان \\
\hline $\mathrm{GN} / \mathrm{m}^{2}$ & 206.8 & Eعامل مرونة الدوار E E E \\
\hline r.p.m & 2000 & السرعة الدور انية N \\
\hline $\mathrm{rad} / \mathrm{s}$ & 209.44 & $\omega=\frac{2 \pi}{60} N$ \\
\hline- & 0.7 & نسبة لاتمركزية العمود و المحمل nn \\
\hline
\end{tabular}

\section{التصرف الحركي للنظام:}

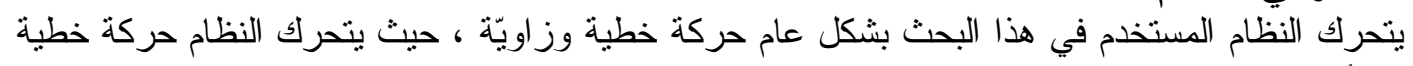

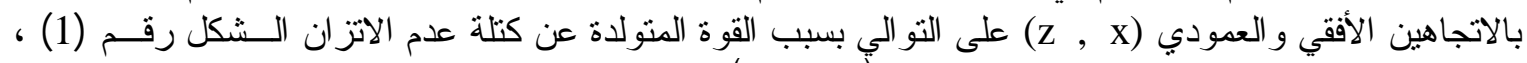

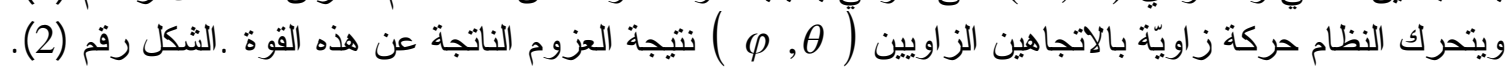

المحامل المقعدية (Journal bearings):

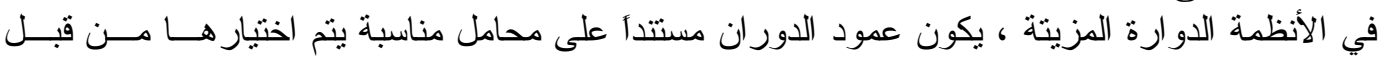

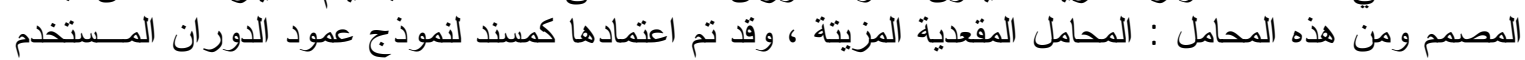
في هذا البحث .

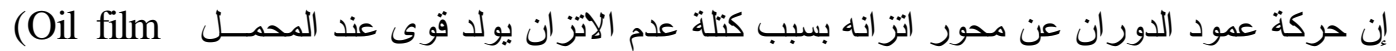

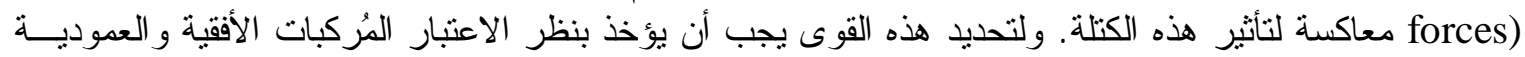

$F_{x}=f_{x}(X, \dot{X}, Z, \dot{Z})$

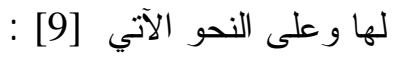

$F_{z}=f_{z}(X, \dot{X}, Z, \dot{Z})$

إذ إن ( $\left.F_{x}, F_{z}\right)$ تعتمدان على عدة عو امل ، منها تصميم المحمل ، و الحمـلـل الـسـاكن ، والــسر عة

الدور انية ، ولزوجة الزيت ـ وبتمثنيل

$-F_{x}=-F_{x o}+K_{x x} \Delta X+K_{x z} \Delta Z+C_{x x} \dot{X}+C_{x z} \dot{Z}+0\left(\Delta X^{2}, \Delta Z^{2}, \Delta \dot{X}^{2}, \Delta \dot{Z}^{2}\right)$

$-F_{z}=-F_{z o}+K_{z x} \Delta X+K_{z z} \Delta Z+C_{z x} \dot{X}+C_{z z} \dot{Z}+0\left(\Delta X^{2}, \Delta Z^{2}, \Delta \dot{X}^{2}, \Delta \dot{Z}^{2}\right)$ 


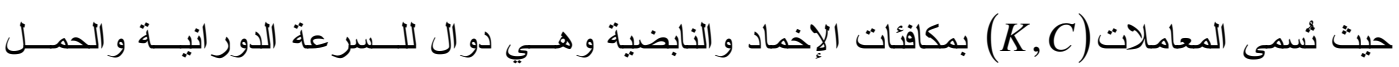

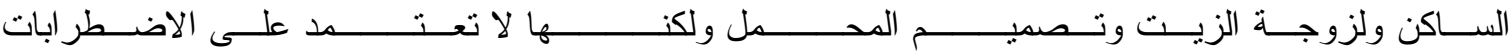

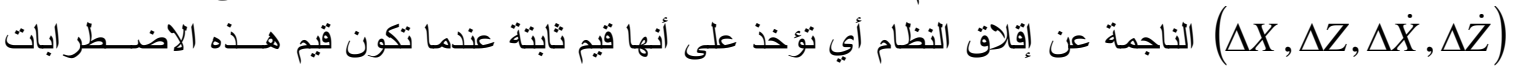

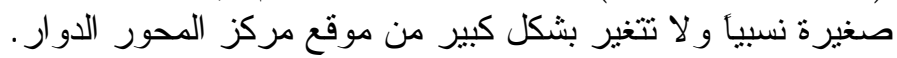

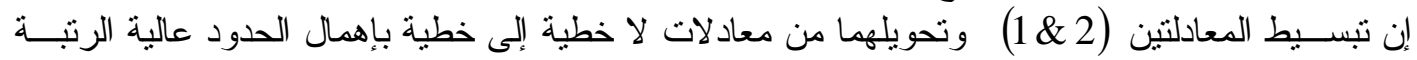

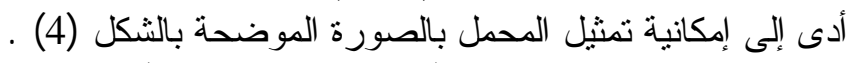
إذ إن المصطلحات $)$

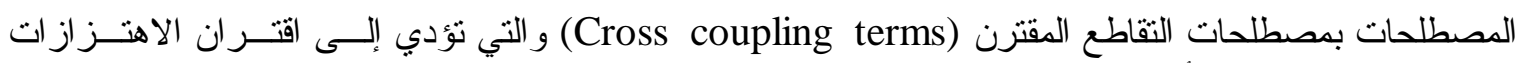

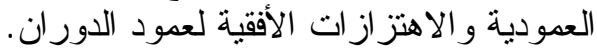
إن اعتماد هذه المصطلحات على السر عة الدور انية جعل اهتزاز ات النظام تعتمد على الحالـــة المـستقرة

للحركة (Steady-state motion)

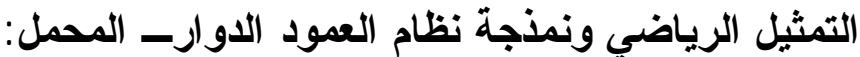

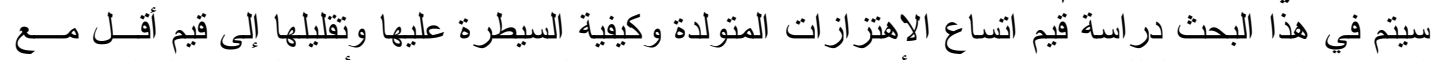

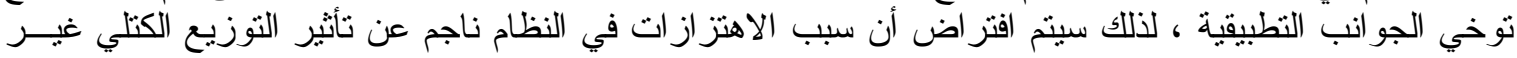

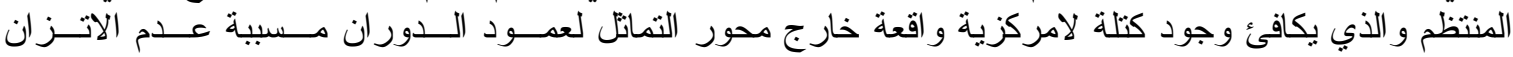

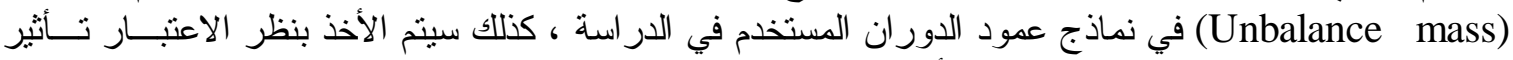

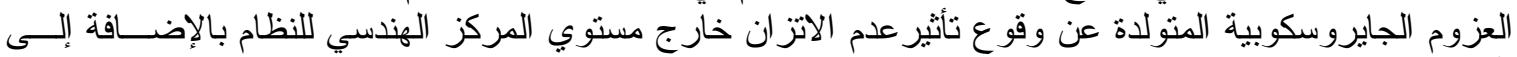

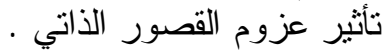

\section{فرضيات نموذج نظام العمود الدوارت الفيز المحل:}

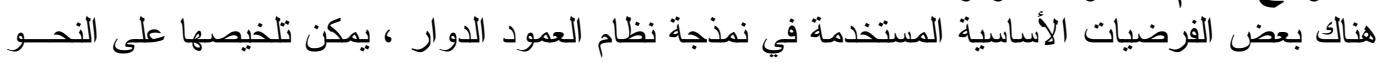
الآتي :

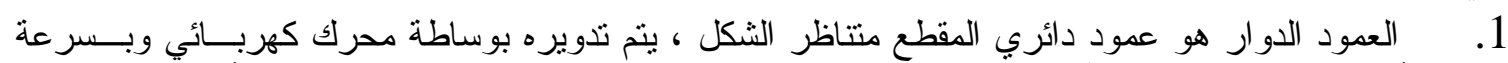

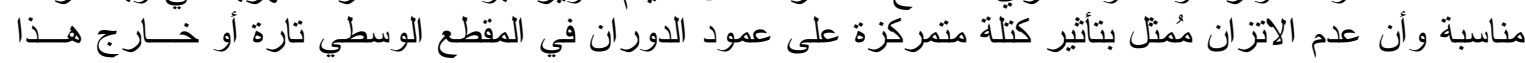

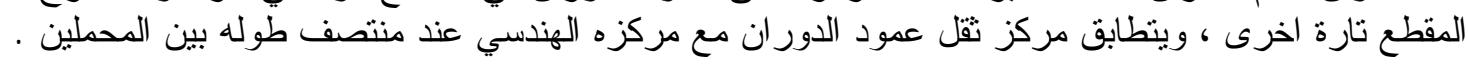

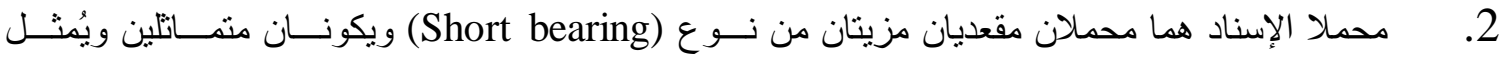

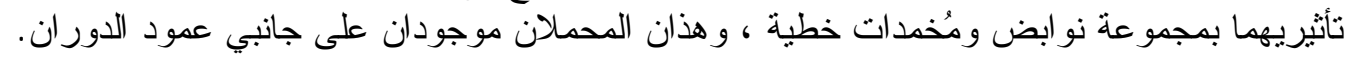

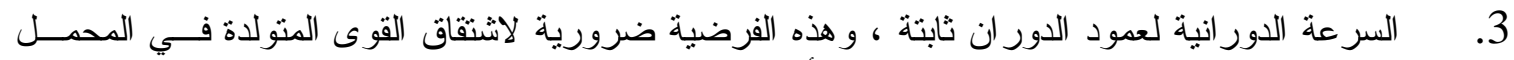

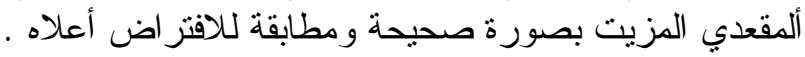

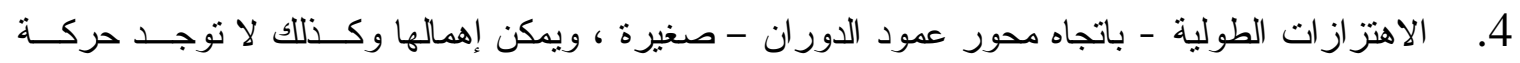

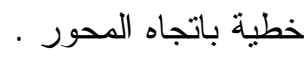

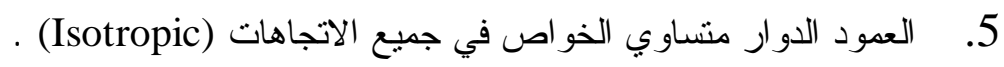

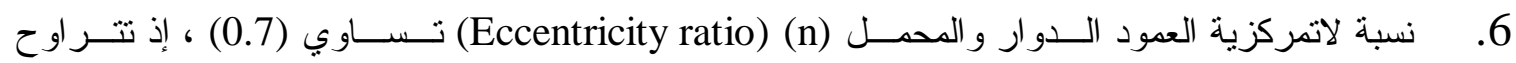

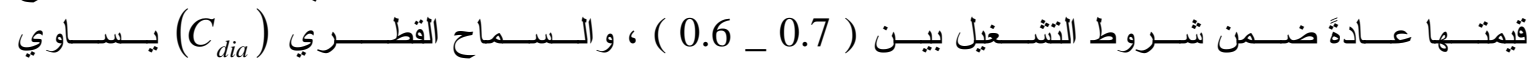

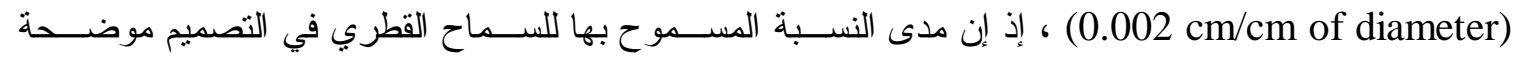

$$
C_{\text {dia }}=0.001 \_0.002 \mathrm{~cm} / \mathrm{cm} \text { of diameter }
$$

على النحو الآتي [10]:

7. مدى اتساع الاهتزازات صغير نسبيا مما يسمح أن تكون القوى المتولدة في طبقة الزيـــت التــي تقابــل العمود خطية وكما ذكر سابقا.

$$
\text { بناء نموذج نظام العمود الدوار - المحمل: }
$$

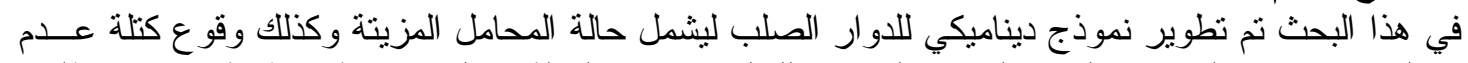

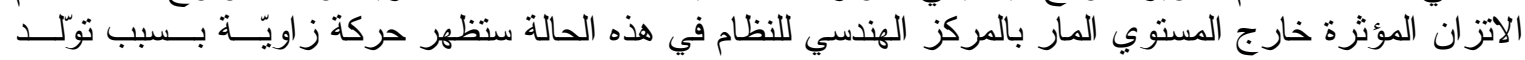




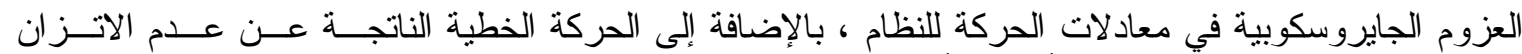

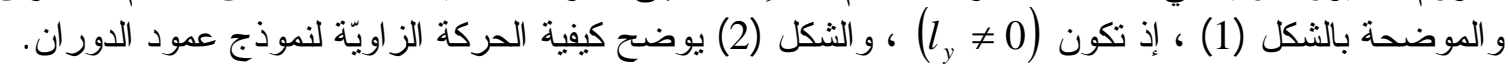

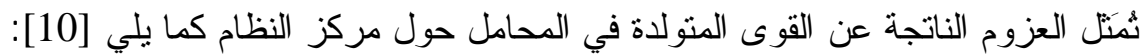
$M_{x}=-2\left[\frac{L^{2}}{4}\left(\theta K_{x x}+\varphi K_{x z}\right)\right]-2\left[\frac{L^{2}}{4}\left(\dot{\theta} C_{x x}+\dot{\varphi} C_{x z}\right)\right]$
$M_{z}=-2\left[\frac{L^{2}}{4}\left(\varphi K_{z z}+\theta K_{z x}\right)\right]-2\left[\frac{L^{2}}{4}\left(\dot{\varphi} C_{z z}+\dot{\theta} C_{z x}\right)\right]$ تصبح معادلات الحركة للدوار على النحو الآتي [4],[11 :

$$
\begin{aligned}
& M \ddot{x}+2 C_{x x} \dot{x}+2 C_{x z} \dot{z}+2 K_{x x} x+2 K_{x z} z=m_{u} l_{z} \omega^{2} \sin (\omega t)+m_{u} l_{x} \omega^{2} \cos (\omega t) \\
& M \ddot{z}+2 C_{z z} \dot{z}+2 C_{z x} \dot{x}+2 K_{z z} z+2 K_{z x} x=-m_{u} l_{x} \omega^{2} \sin (\omega t)+m_{u} l_{z} \omega^{2} \cos (\omega t)
\end{aligned}
$$

$I_{t} \ddot{\theta}-I_{p} \dot{\varphi} \omega+\frac{L^{2}}{2}\left(\theta K_{x x}+\varphi K_{x z}\right)+\frac{L^{2}}{2}\left(\dot{\theta} C_{x x}+\dot{\varphi} C_{x z}\right)=-m_{u} l_{y} l_{x} \omega^{2} \sin (\omega t)+m_{u} l_{y} l_{z} \omega^{2} \cos (\omega t)$ $I_{t} \ddot{\varphi}+I_{p} \dot{\theta} \omega+\frac{L^{2}}{2}\left(\varphi K_{z z}+\theta K_{z x}\right)+\frac{L^{2}}{2}\left(\dot{\varphi} C_{z z}+\dot{\theta} C_{z x}\right)=-m_{u} l_{y} l_{z} \omega^{2} \sin (\omega t)-m_{u} l_{y} l_{x} \omega^{2} \cos (\omega t)$ وعند تحويل معادلات الحركة هذه إلى صيغة فضاء الحالة ، ( صيغة

$\left[\begin{array}{c}\dot{x} \\ \dot{z} \\ \dot{\theta} \\ \dot{\varphi} \\ \ddot{x} \\ \ddot{z} \\ \ddot{\theta} \\ \ddot{\varphi}\end{array}\right]=\left[\begin{array}{cccccccc}0 & 0 & 0 & 0 & 1 & 0 & 0 & 0 \\ 0 & 0 & 0 & 0 & 0 & 1 & 0 & 0 \\ 0 & 0 & 0 & 0 & 0 & 0 & 1 & 0 \\ 0 & 0 & 0 & 0 & 0 & 0 & 0 & 1 \\ \frac{-2 K_{x x}}{M} & \frac{-2 K_{x z}}{M} & 0 & 0 & \frac{-2 C_{x x}}{M} \frac{-2 C_{x z}}{M} & 0 & 0 \\ \frac{-2 K_{z x}}{M} & \frac{-2 K_{z z}}{M} & 0 & 0 & \frac{-2 C_{z x}}{M} \frac{-2 C_{z z}}{M} & 0 & 0 \\ 0 & 0 & \frac{-L^{2} K_{x x}}{2 I_{t}} \frac{-L^{2} K_{x z}}{2 I_{t}} & 0 & 0 & \frac{-L^{2} C_{x x}}{2 I_{t}} & \frac{I_{p} \omega}{I_{t}}-\frac{L^{2} C_{x z}}{2 I_{t}} \\ 0 & 0 & \frac{-L^{2} K_{z x}}{2 I_{t}} & \frac{-L^{2} K_{z z}}{2 I_{t}} & 0 & 0 & \frac{-I_{p} \omega}{I_{t}}-\frac{L^{2} C_{z x}}{2 I_{t}} & \frac{-L^{2} C_{z z}}{2 I_{t}}\end{array}\right]\left[\begin{array}{c}x \\ z \\ \theta \\ \varphi \\ \dot{x} \\ \dot{z} \\ \dot{\theta} \\ \dot{\varphi}\end{array}\right]+\left[\begin{array}{ccc}0 & 0 \\ 0 & 0 \\ 0 & 0 \\ 0 & 0 \\ \frac{m_{u} l_{z}}{M} & \frac{m_{u} l_{x}}{M} \\ \frac{-m_{u} l_{x}}{M} & \frac{m_{u} l_{z}}{M} \\ \frac{-m_{u} l_{x} l_{y}}{I_{t}} & \frac{m_{u} l_{z} l_{y}}{I_{t}} \\ \frac{-m_{u} l_{z} l_{y}}{I_{t}} & \frac{-m_{u} l_{x} l_{y}}{I_{t}}\end{array}\right]\left[\begin{array}{c}\omega^{2} \sin (\omega t) \\ \omega^{2} \cos (\omega t)\end{array}\right]$

$\mathrm{m}$ الد :

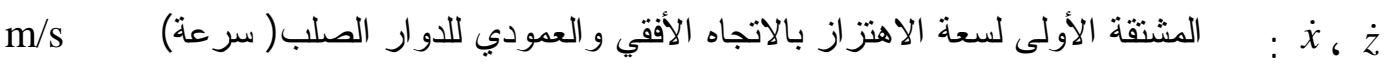

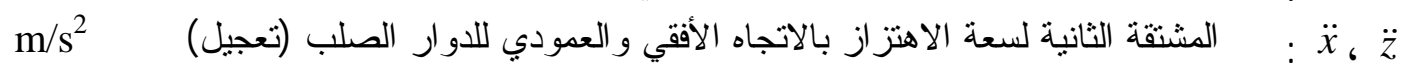

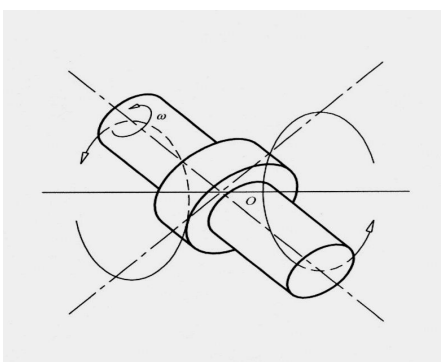

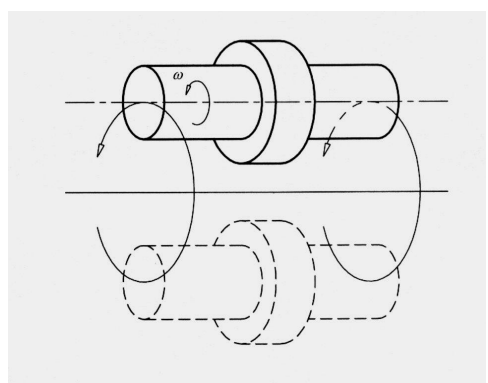

الشكل (1): الحركة الانتقالية لعمود الدور ان الصلب. 


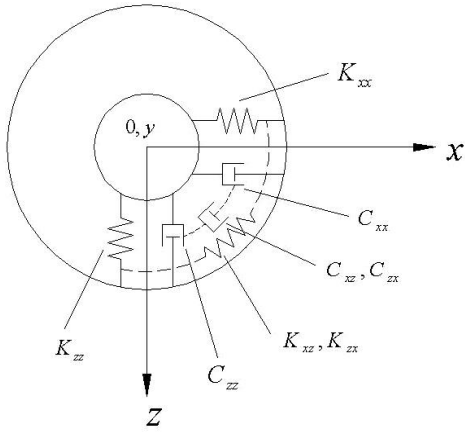

الثكل (4): النموذج الخطي للمحمل المقعدي.

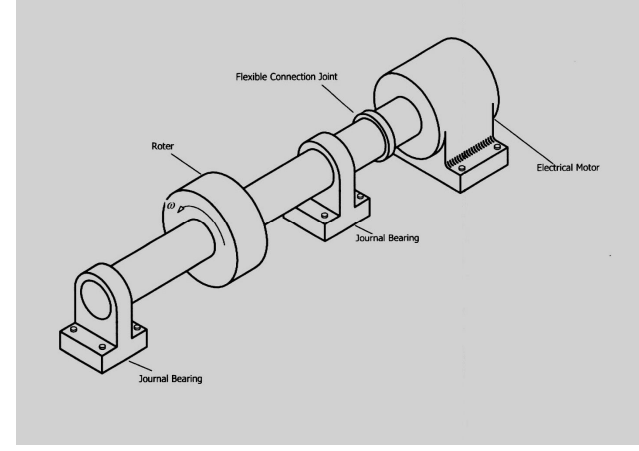

الثكل (3): الشكل العام لنظام العمود الدوار - المحمل.

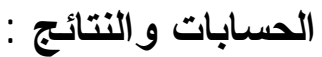

إن السيطرة على النظام تمت باستخدام تقنيتي تعيين القطب و السيطرة المثالية. و إن القوة يــتم تـسليطها

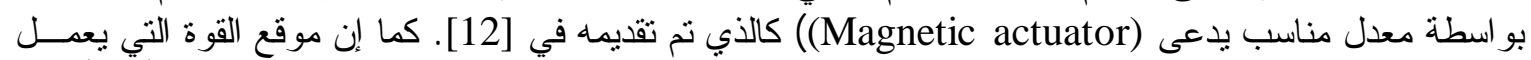

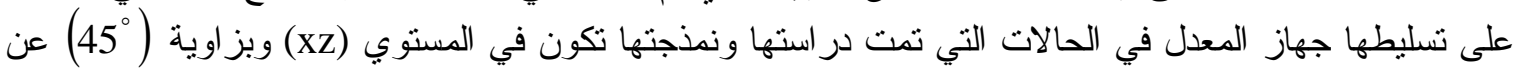

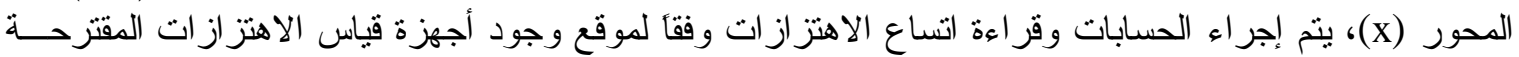

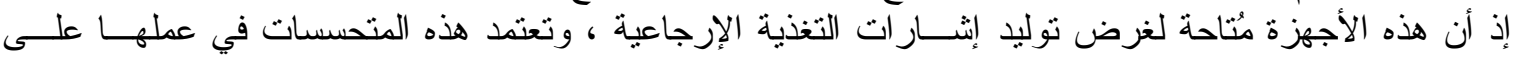

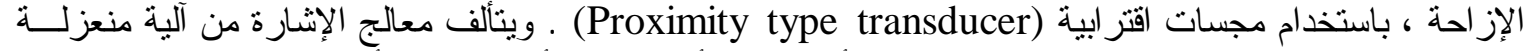

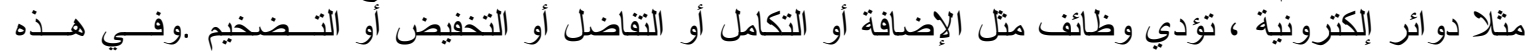

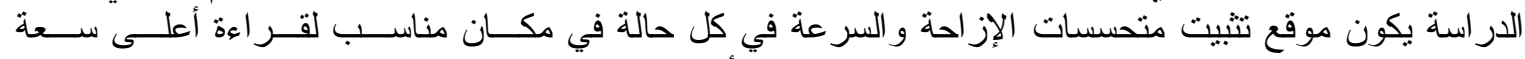

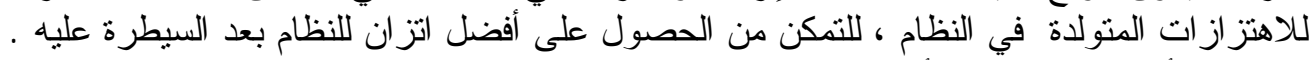

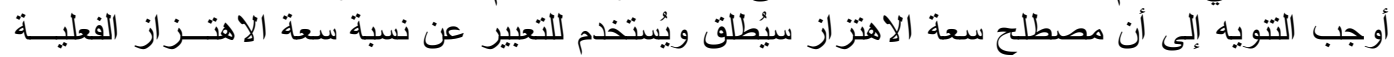

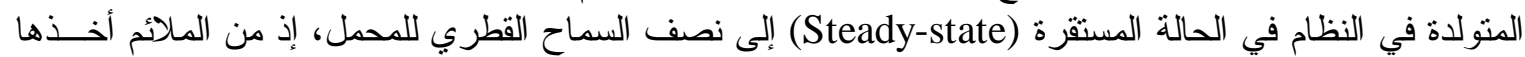

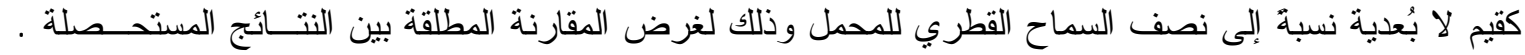

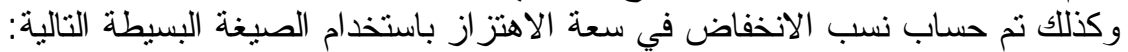

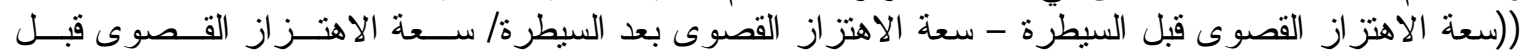
السيطرة) × 100

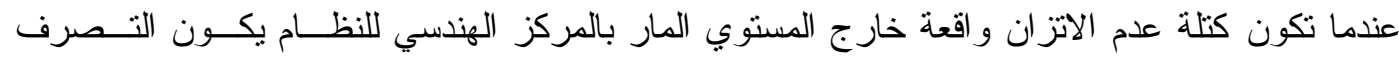

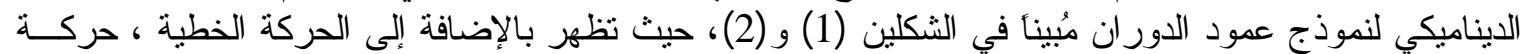

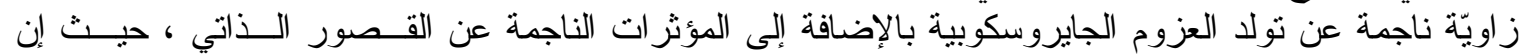

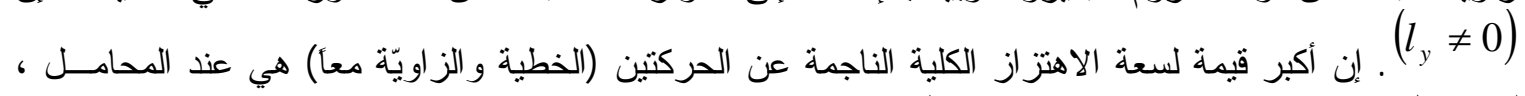

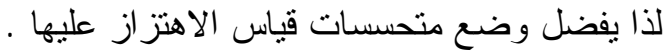

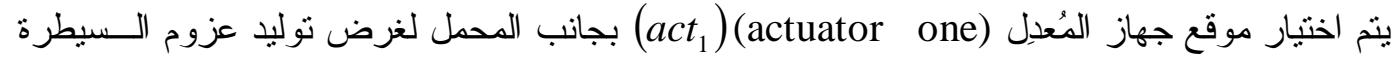

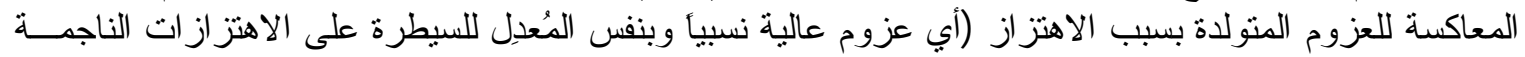

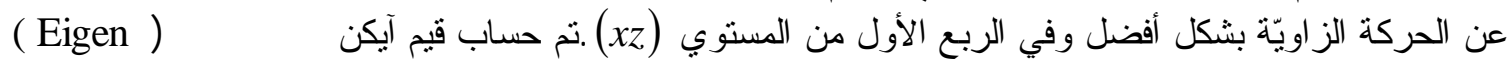

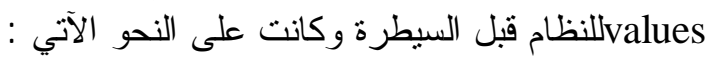
$s_{1}=-3224.7 \quad$ ، $s_{2,3}=-92.767 \mp 184.8 i \quad$ ، $s_{4}=-364.44$ $s_{5}=-15363 \quad$ ، $s_{6}=-1777.3 \quad$ ، $s_{7,8}=-131.38 \mp 147.84 i$

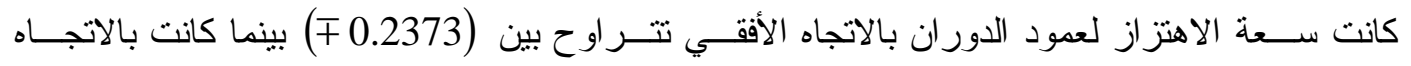

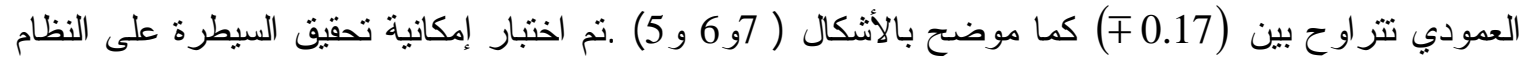
$P=\left[\begin{array}{llllllll}B & A B & A^{2} B & . & . & . & A^{m-1} B\end{array}\right]$ باستخدام مصفوفة تحقيق السبطرة P و والممنلة بالعلاقة آلاتية: 

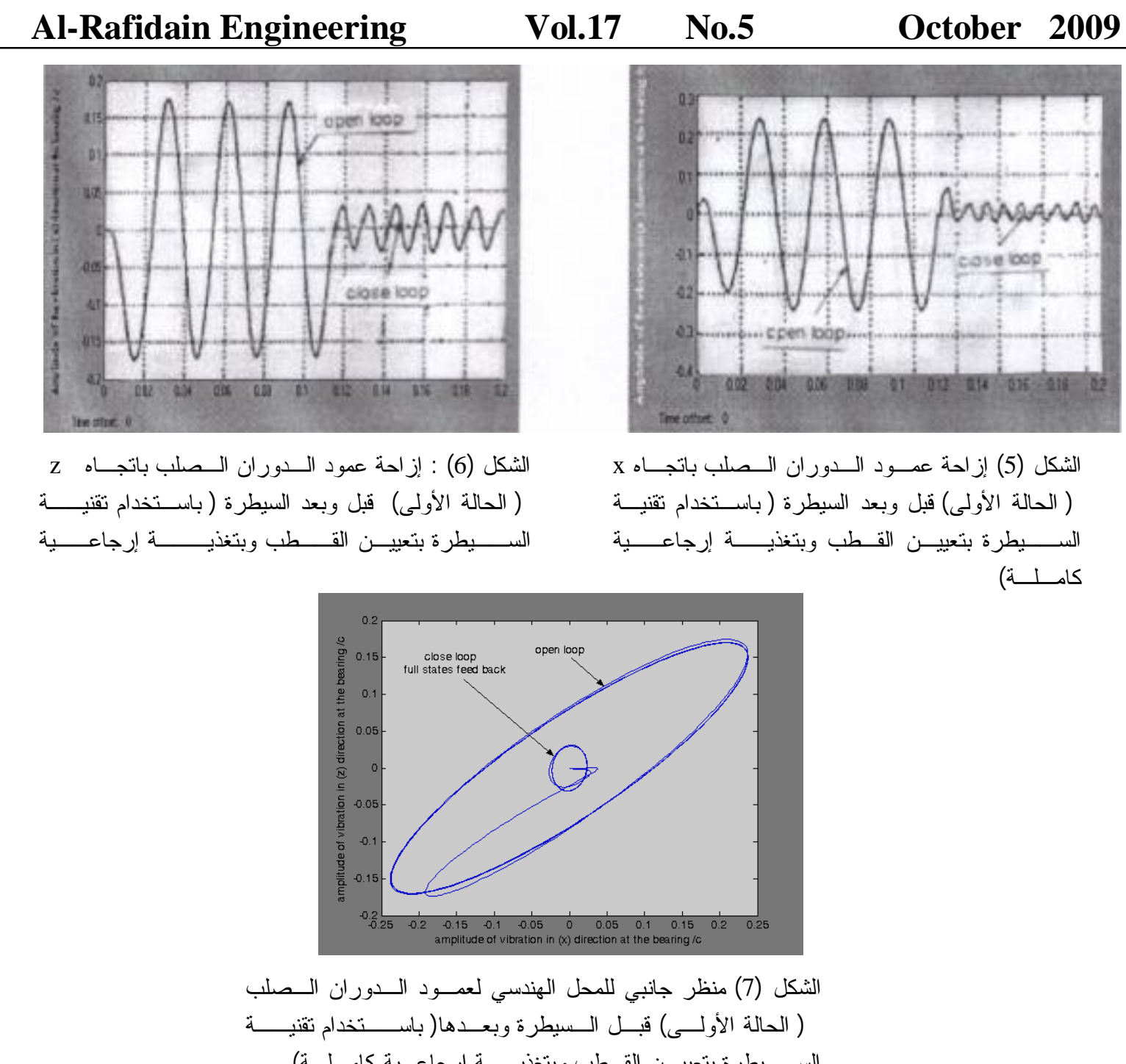

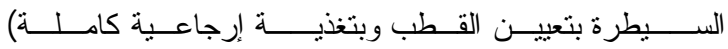

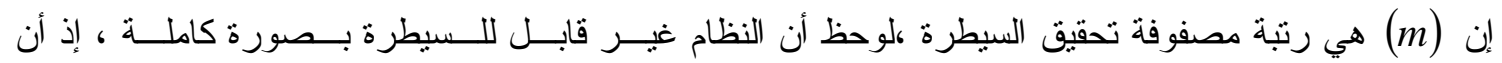
(m=3) متغير ات الحالة لتحقيق السيطرة الكاملة على النظف النظام.

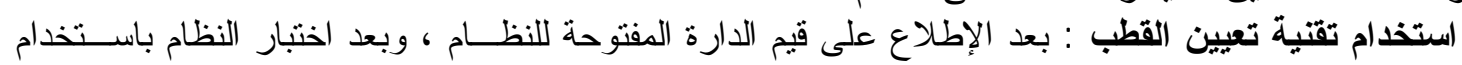

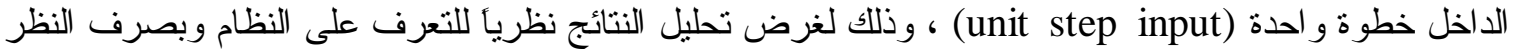

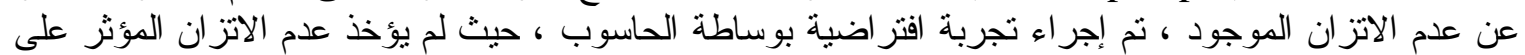
المنظومة ، وروجد أن الأقطاب (

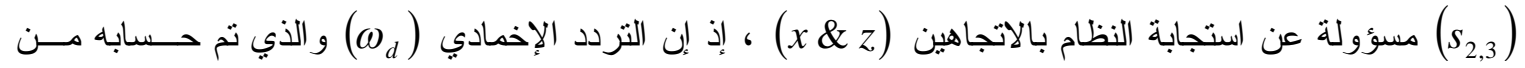

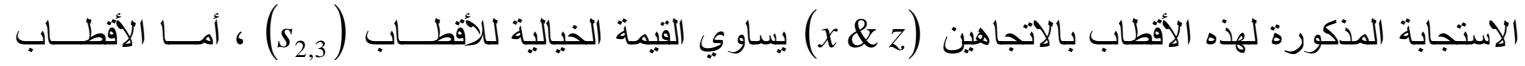

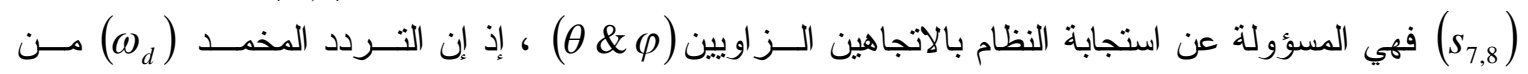
الاستجابة للنظام بالاتجاهين (

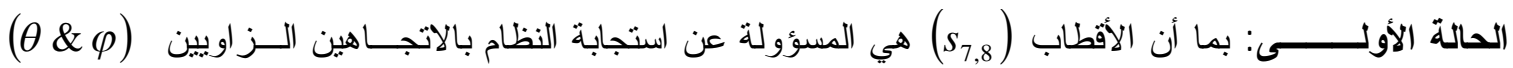

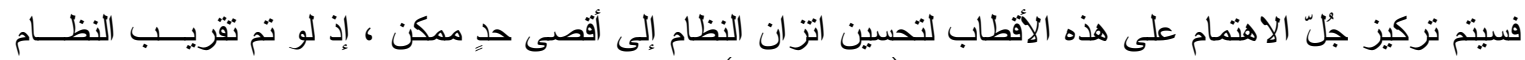

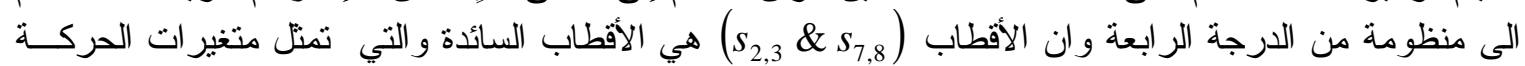

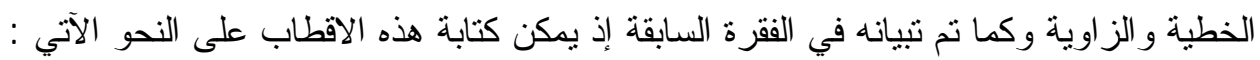

$$
s_{2,3}=-\xi_{1} \omega_{n r_{1}} \mp \sqrt{1-\xi_{1}^{2}} \omega_{n r_{1}} i \quad \text { ، } \quad s_{7,8}=-\xi_{2} \omega_{n r_{2}} \mp \sqrt{1-\xi_{2}^{2}} \omega_{n r_{2}} i
$$


علما ان الجزء الخيالي من هذا الترميز الطوري يمثل التردد الاخمادي للمنظومة في حالة تصرفها بشكل انتقـالي

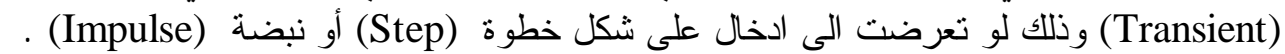

من ذلك يمكن معرفة قيمتي التزدد الطبيعي (

$$
\begin{aligned}
& \xi_{1}=0.449 \quad, \quad \omega_{n r_{1}}=206.78 \quad \mathrm{rad} / \mathrm{sec} \\
& \xi_{2}=0.6643 \quad, \quad \omega_{n r_{2}}=197.78 \quad \mathrm{rad} / \mathrm{sec}
\end{aligned}
$$

له ، وذللك على النحو الآتي:

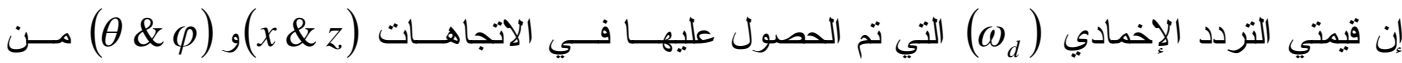

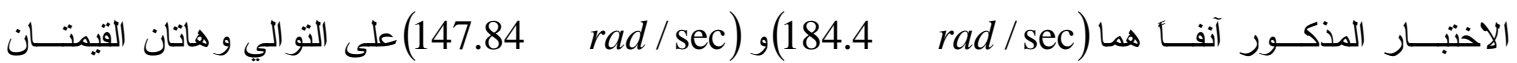

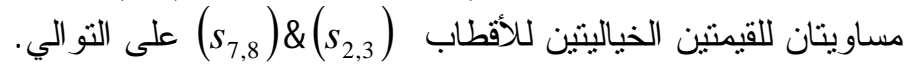

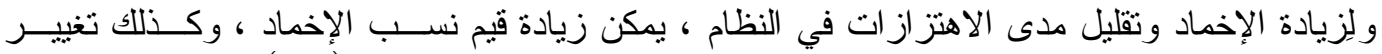

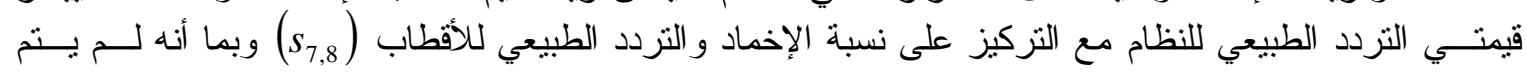

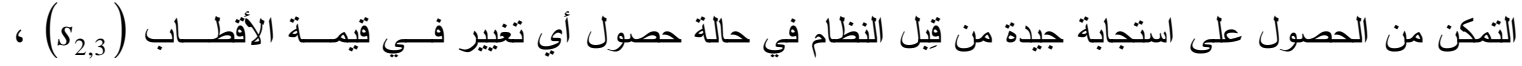

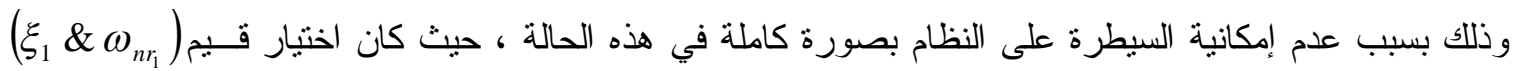

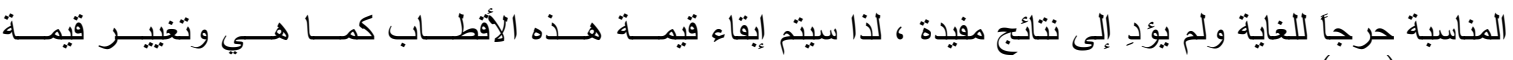
الأقطاب (s,8) فقط وذللك على النحو الآتي : $\xi_{2}=0.9 \quad, \quad \omega_{n r_{2}}=197.78 \times 2=791.12 \quad \mathrm{rad} / \mathrm{sec}$ و عند ذالك تصبح الأفطاب (s,8) $s_{7,8}=-712 \mp 344.84 i$

وبذلك تكون الأقطاب المختارة للارارة المغلقـــة موضحة على النحو الآتي :

$S_{1}=-3224.7$ ، $s_{2,3}=-94 \mp 184 i$ 。 $s_{4}=-364.44$

$s_{7,8}=-712 \mp 344.84 i$ $s_{6}=-1777.3$ ، $s_{5}=-15363$

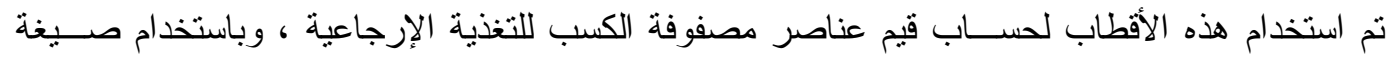

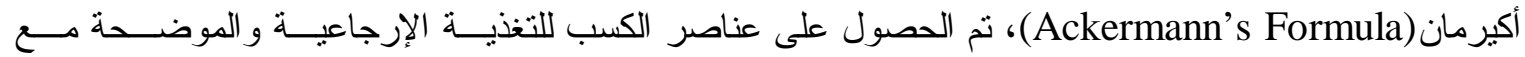

\begin{tabular}{|c|c|c|c|c|c|c|c|c|}
\hline state & $x$ & $z$ & $\theta$ & $\varphi$ & $\dot{x}$ & $\dot{z}$ & $\dot{\theta}$ & $\dot{\varphi}$ \\
\hline $\begin{array}{c}\text { gain } \\
K\end{array}$ & $\begin{array}{c}\times 1.12 \\
10^{6}\end{array}$ & $\begin{array}{c}\times 3.18 \\
10^{6}\end{array}$ & $\begin{array}{c}- \\
\times 3.12 \\
10^{6}\end{array}$ & $\begin{array}{c}\times 7.22 \\
10^{7}\end{array}$ & $\begin{array}{c}2011 . \\
9\end{array}$ & 1609.8 & 7563 & $\begin{array}{c}7574 . \\
7\end{array}$ \\
\hline
\end{tabular}
متغير ات الحالة الخاصة بها على النحو الآتي :

أصبحت نسبة الانخفاض في سعة الاهنز از بالاتجاه الأفقي تساوي (90\%) وبالاتجاه العمودي تساوي (81.6\%)

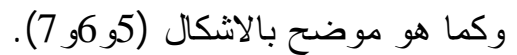

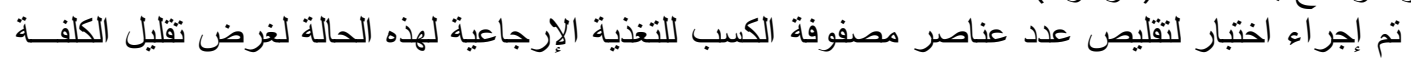

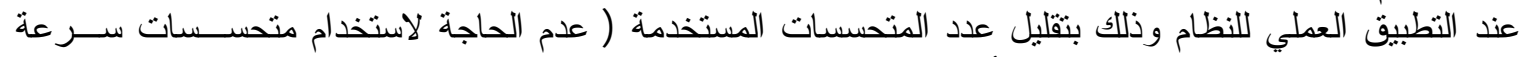

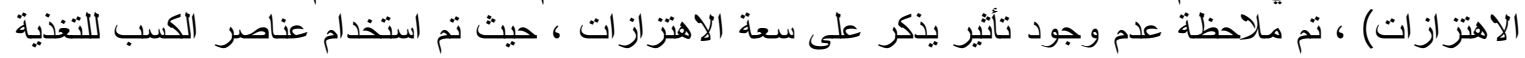
الإرجاعية الموضحة مع مت متغير ات الحالة الخاصة بها على على النحو الآتي:

\begin{tabular}{|c|c|c|c|c|}
\hline state & $x$ & $z$ & $\theta$ & $\varphi$ \\
\hline Gain $K$ & $1.12 \times 10^{6}$ & $3.18 \times 10^{6}$ & $-3.12 \times 10^{6}$ & $7.22 \times 10^{7}$ \\
\hline
\end{tabular}

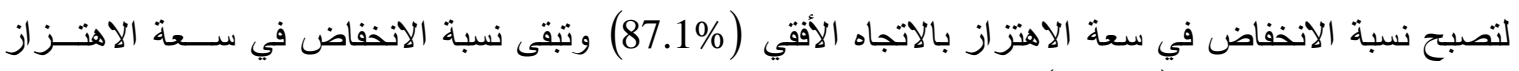

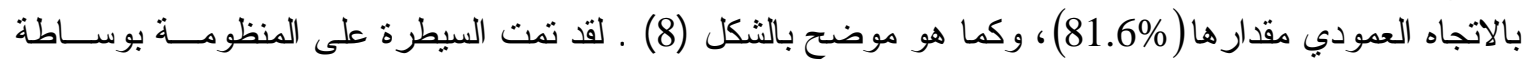




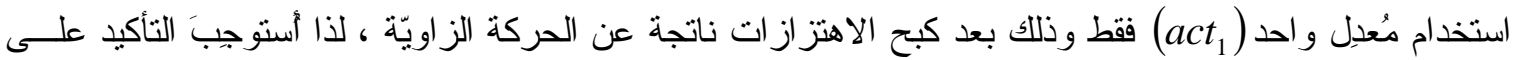

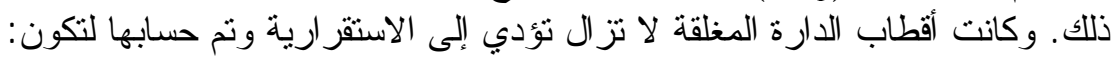

$s_{1}=-3221.2$ ، $\quad s_{2,3}=-94.222 \mp 184.08 i \quad s_{4}=-357.88$

$s_{5}=-15156 \quad$ ، $\quad s_{6}=-556.85 \quad$ ، $\quad s_{7,8}=-848.55 \mp 1157.9 i$

إن النتائج المستحصلة من الأقطاب المختارة قد عملت على تقليل الاهنزاز ات إلى إلى حدٍ كبير, ، وظلّ النظام

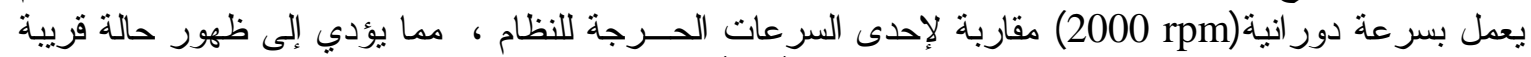

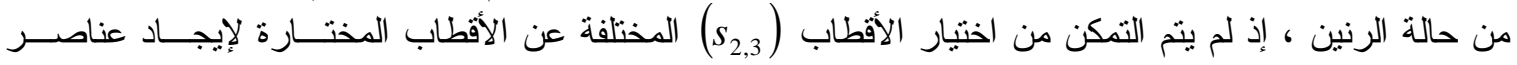

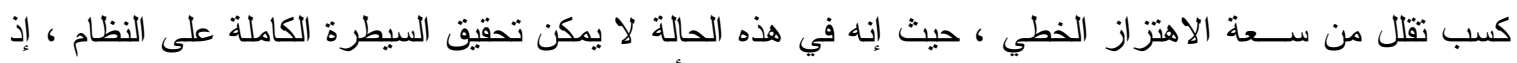

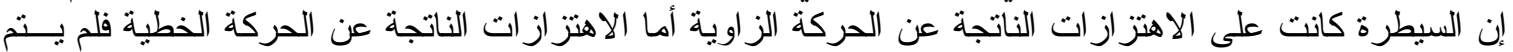

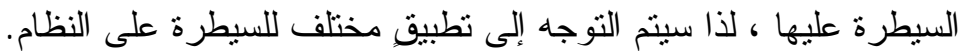

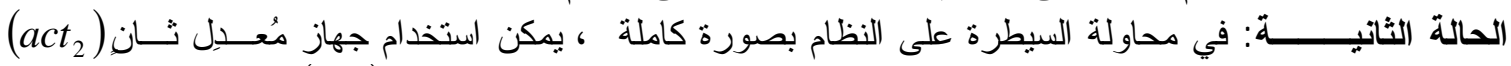

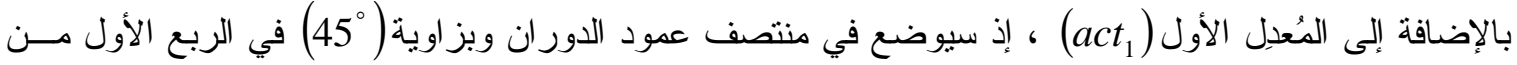

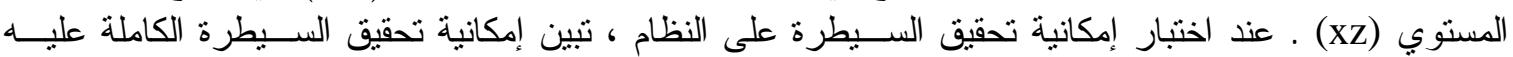

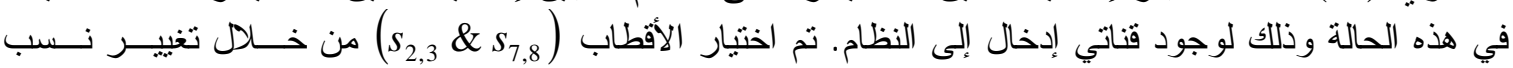
الإخماد و التزدد الطبيعي للنظام وذللك على النحو الآتي:

$\xi_{1}=0.85 \quad, \quad \omega_{n r_{1}}=206.78 \times 4=827.12 \quad \mathrm{rad} / \mathrm{sec}$ $\xi_{2}=0.9 \quad, \quad \omega_{n r_{2}}=197.78 \times 8=1582.24 \quad \mathrm{rad} / \mathrm{sec}$

تصبح قيم الأقطاب المختارة للارة المغلقة موضحة على النحو الآتي :

$s_{1}=-3224.7 \quad$ ، $s_{2,3}=-703.05 \mp 435.72 i \quad$ ، $\quad s_{4}=-364.44$

$s_{5}=-15363 \quad$ ، $s_{6}=-1777.3 \quad$ ، $s_{7,8}=-1424 \mp 689.68 i$

عند استخدام هذه الأقطاب لإيجاد عناصر مصفوفة الكسب للتغذية الإرجاعية باســتخدام صــيغة أكيرمان ، كانت مع متغيرات الحالة الخاصة بهاب على الإنى النحو الآتي :

\begin{tabular}{|c|c|c|c|c|c|c|c|c|}
\hline state & $x$ & $z$ & $\theta$ & $\varphi$ & $\dot{x}$ & $\dot{z}$ & $\dot{\theta}$ & $\dot{\varphi}$ \\
\hline$(K)_{a c t_{1}}$ & $\begin{array}{c}\times 5.584 \\
10^{6}\end{array}$ & $\begin{array}{c}- \\
\times 1.400 \\
10^{7} \\
\end{array}$ & $\begin{array}{c}\times 7.571 \\
10^{6}\end{array}$ & $\begin{array}{c}\times 5.806 \\
10^{7}\end{array}$ & $\begin{array}{c}2738 \\
.3\end{array}$ & -45842 & $\begin{array}{c}8954 . \\
3\end{array}$ & 6902 \\
\hline$(K)_{a c t_{2}}$ & $\begin{array}{c}- \\
\times 1.85 \\
10^{7} \\
\end{array}$ & $\begin{array}{c}\times 6.972 \\
10^{8}\end{array}$ & $\begin{array}{c}\times 3.669 \\
10^{7}\end{array}$ & $\begin{array}{c}- \\
\times 1.260 \\
10^{8}\end{array}$ & $\begin{array}{c}6232 \\
7\end{array}$ & $\begin{array}{c}\times 2.422 \\
10^{5}\end{array}$ & $\begin{array}{c}524.4 \\
5 \\
\end{array}$ & $\begin{array}{c}- \\
1513 \\
2 \\
\end{array}$ \\
\hline
\end{tabular}

إن نســبة الانخفاض في سعة الاهتزاز بالاتجاه الأفقي بلغت (86.1\%) ، ونسبة انخفاض في سعة الاهتز از

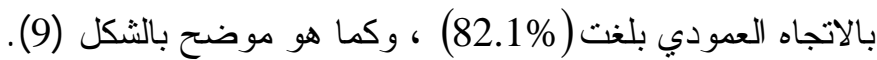

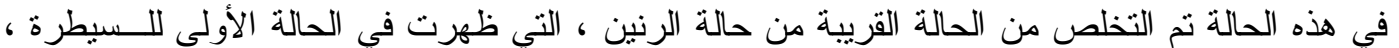

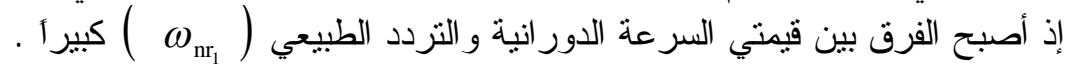

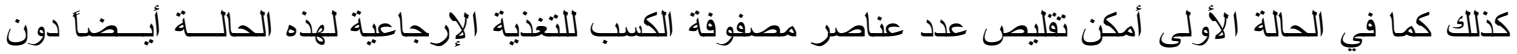

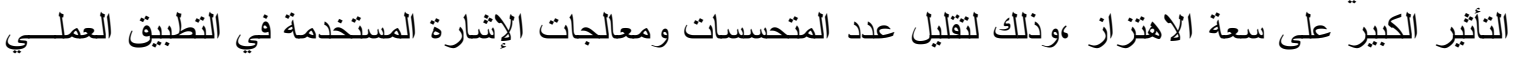

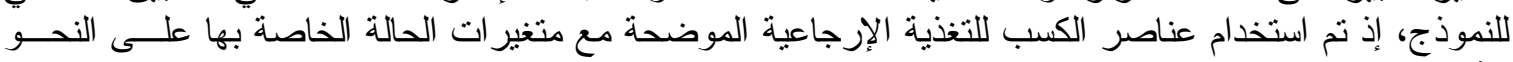
الآتي :

\begin{tabular}{|c|c|c|c|c|}
\hline & $x$ & $z$ & $\theta$ & $\varphi$ \\
\hline$(K)_{\text {act }_{1}}$ & $5.5841 \times 10^{6}$ & $-1.4002 \times 10^{7}$ & $7.5705 \times 10^{6}$ & $5.8061 \times 10^{7}$ \\
\hline$(K)_{\text {act }_{2}}$ & $-1.853 \times 10^{7}$ & $6.9719 \times 10^{8}$ & $3.6688 \times 10^{7}$ & $-1.2606 \times 10^{8}$ \\
\hline
\end{tabular}




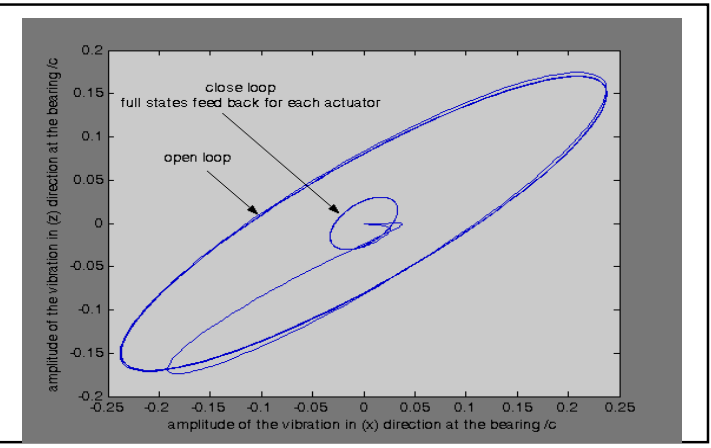

الثنكل (9) منظر جانبي للمحل الهندسي لـعمود الــدوران

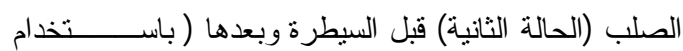

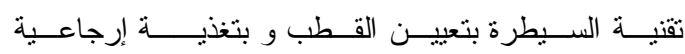
كامـلة )

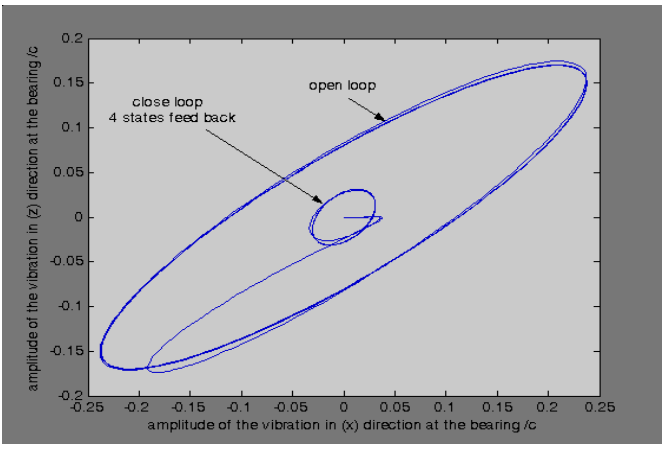

الثكل (8) منظر جانبي للمحل الهندسي لعـــود الــدوران

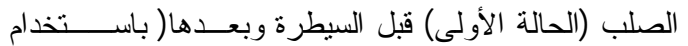

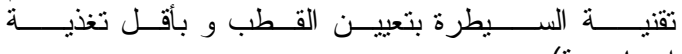

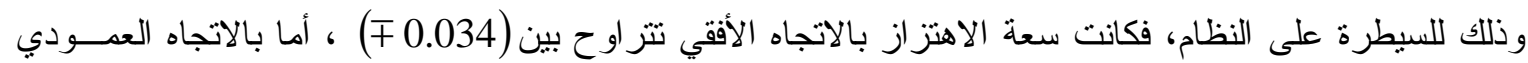

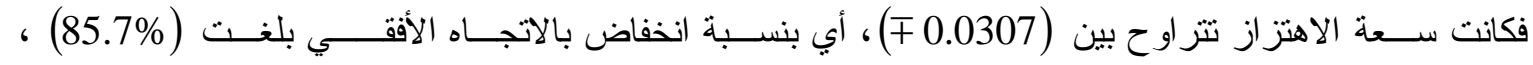

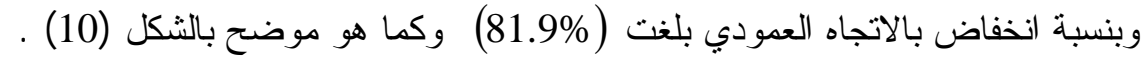

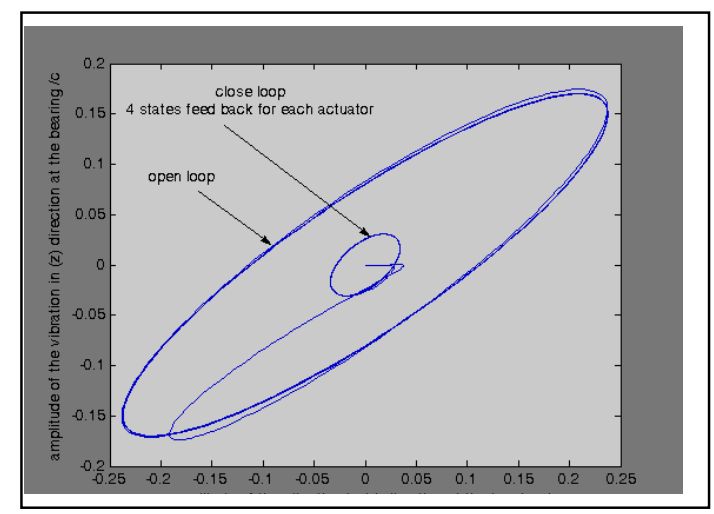

الثكل (10) منظر جانبي للمحل الهندسي لــعمود الدور ان الصلب بانب

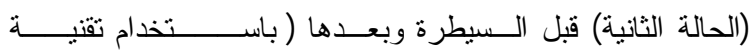

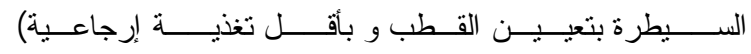

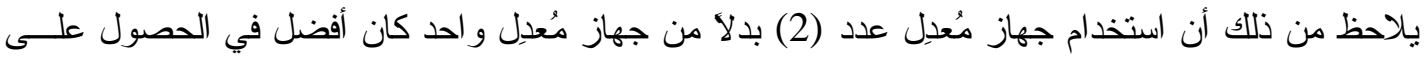

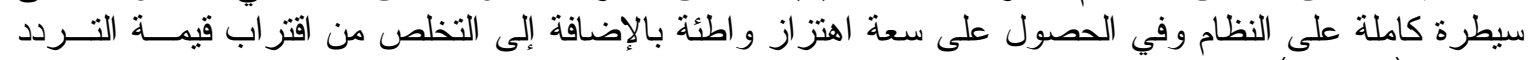
الطبيعي (

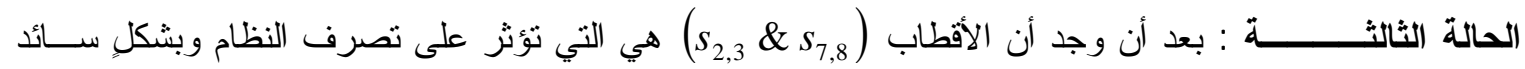

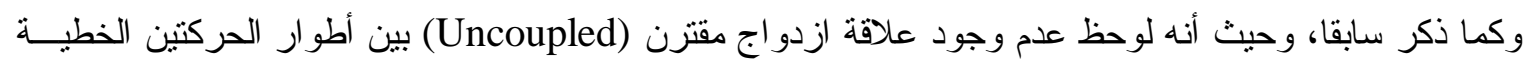

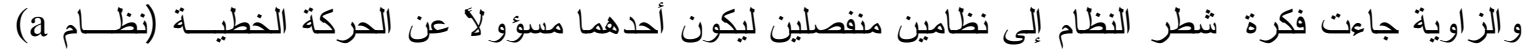

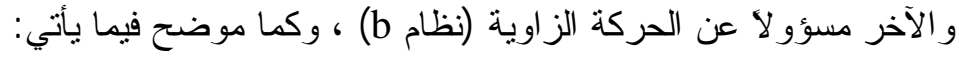

$$
\left[\begin{array}{c}
\dot{x} \\
\dot{z} \\
\ddot{x} \\
\ddot{z}
\end{array}\right]=\left[\begin{array}{cccc}
0 & 0 & 1 & 0 \\
0 & 0 & 0 & 1 \\
\frac{-2 K_{x x}}{M} & \frac{-2 K_{x z}}{M} & \frac{-2 C_{x x}}{M} & \frac{-2 C_{x z}}{M} \\
\frac{-2 K_{z x}}{M} & \frac{-2 K_{z z}}{M} & \frac{-2 C_{z x}}{M} & \frac{-2 C_{z z}}{M}
\end{array}\right]\left[\begin{array}{c}
x \\
z \\
\dot{x} \\
\dot{z}
\end{array}\right]+\left[\begin{array}{cc}
0 & 0 \\
0 & 0 \\
\frac{m_{u} l_{z}}{M} & \frac{m_{u} l_{x}}{M} \\
-\frac{m_{u} l_{x}}{M} & \frac{m_{u} l_{z}}{M}
\end{array}\right]\left[\begin{array}{c}
\omega^{2} \sin (\omega t) \\
\omega^{2} \cos (\omega t)
\end{array}\right]
$$

\section{•}




$$
\left[\begin{array}{c}
\dot{\theta} \\
\dot{\varphi} \\
\ddot{\theta} \\
\ddot{\varphi}
\end{array}\right]=\left[\begin{array}{cccc}
0 & 0 & 1 & 0 \\
0 & 0 & 0 & 1 \\
\frac{-L^{2} K_{x x}}{2 I_{t}} & \frac{-L^{2} K_{x z}}{2 I_{t}} & \frac{-L^{2} C_{x x}}{2 I_{t}} & \frac{I_{p}}{I_{t}} \omega-\frac{L^{2} C_{x z}}{2 I_{t}} \\
\frac{-L^{2} K_{z x}}{2 I_{t}} & \frac{-L^{2} K_{z z}}{2 I_{t}} & -\frac{I_{p}}{I_{t}} \omega-\frac{L^{2} C_{z x}}{2 I_{t}} & \frac{-L^{2} C_{z z}}{2 I_{t}}
\end{array}\right]\left[\begin{array}{c}
\theta \\
\varphi \\
\dot{\theta} \\
\dot{\varphi}
\end{array}\right]+\left[\begin{array}{cc}
0 & 0 \\
0 & 0 \\
-\frac{m_{u} l_{x} l_{y}}{I_{t}} & \frac{m_{u} l_{z} l_{y}}{I_{t}} \\
-\frac{m_{u} l_{z} l_{y}}{I_{t}} & -\frac{m_{u} l_{x} l_{y}}{I_{t}}
\end{array}\right]\left[\begin{array}{c}
\omega^{2} \sin (\omega t) \\
\omega^{2} \cos (\omega t)
\end{array}\right]
$$

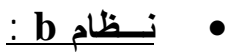

في هذه الحالة تم استخدام المُعدِل (act

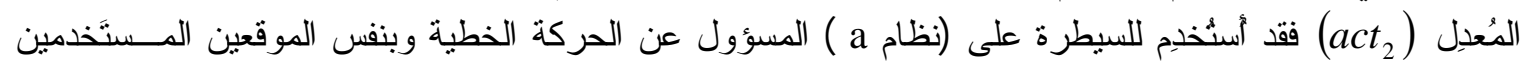

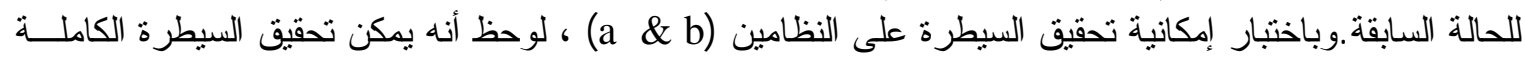

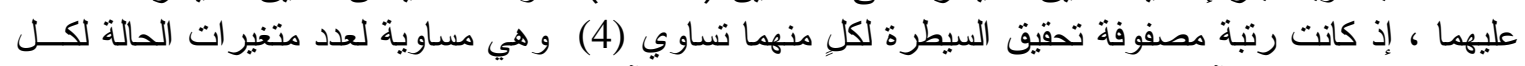

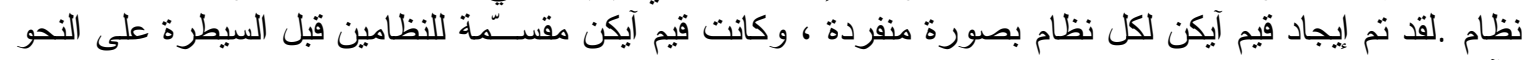

$s_{1}=-3224.7 \quad s_{2,3}=-92.767 \mp 184.8 i$ قيميم آيكن لنظام (a) ) قبل السبطرة : الآتي:

$$
s_{5}=-15363 \quad \text { ، } \quad s_{6}=-1777.3 \quad \text { ، } \quad s_{7,8}=-131.38 \mp 147.84 i
$$

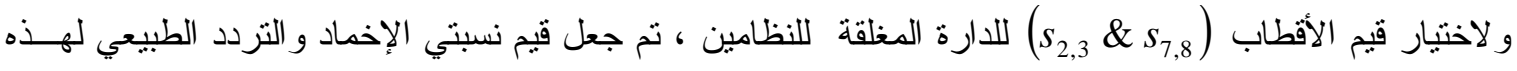

$\xi_{1}=0.85 \quad, \quad \omega_{n r_{1}}=206.78 \times 4=827.12 \quad \mathrm{rad} / \mathrm{sec}$.

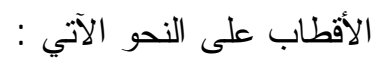

$\xi_{2}=0.9 \quad, \quad \omega_{n r_{2}}=197.78 \times 4=791.12 \quad \mathrm{rad} / \mathrm{sec}$

وبذلك أصبحت قيم الأقطاب (نظام

$s_{2,3}=-703.05 \mp 435.7 i \quad$ ، $\quad s_{7,8}=-712 \mp 344.84 i$

عند ذلك تكون الأقطاب المختارة للسيطرة على النظامين موضحة على النحو الآتي :

$$
\begin{aligned}
& s_{1}=-3224.7 \quad \text { ، } \quad s_{2,3}=-703.05 \mp 435.7 i \quad \text { ، } \quad s_{4}=-364.44
\end{aligned}
$$

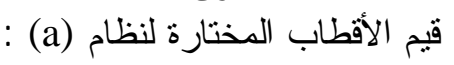

$$
\begin{aligned}
& \text { قيم الأقطاب المختارة لنظام (b): } \\
& s_{5}=-15363 \quad \text { ، } \quad s_{6}=-1777.3 \\
& s_{7,8}=-712 \mp 344.84 i
\end{aligned}
$$

عند استخدام هذه الأقطاب تم الحصول على مصفوفتي الكسب للتغذية الإرجاعية للنظــامين وباســتخدام

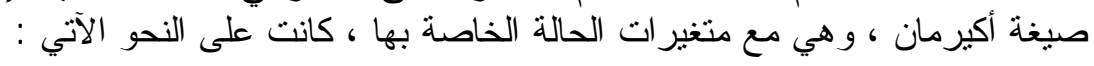

عناصر الكسب لنظام ( a ) :

\begin{tabular}{|c|c|c|c|c|}
\hline State & $x$ & $z$ & $\dot{x}$ & $\dot{z}$ \\
\hline$(K)_{\text {act }_{2}}$ & $4.3059 \times 10^{7}$ & $1.1514 \times 10^{8}$ & $8.0276 \times 10^{4}$ & $6.0735 \times 10^{4}$ \\
\hline
\end{tabular}

عناصر الكسب لنظام ( b ) : ( )

\begin{tabular}{|c|c|c|c|c|}
\hline State & $\theta$ & $\varphi$ & $\dot{\theta}$ & $\dot{\varphi}$ \\
\hline$(K)_{a t_{1}}$ & $-3.326 \times 10^{6}$ & $7.4432 \times 10^{7}$ & $7.7361 \times 10^{3}$ & $7.7876 \times 10^{3}$ \\
\hline
\end{tabular}


باستخدام هاتين المصفوفتين للسيطرة على النظام الغلي ، كانت سعة الاهتزاز في الاتجاه الأفقي تتزاوح

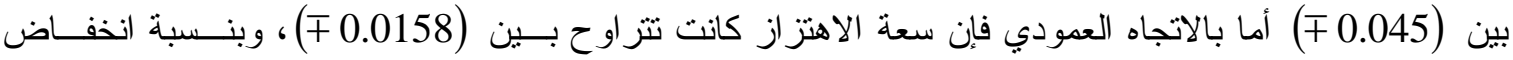

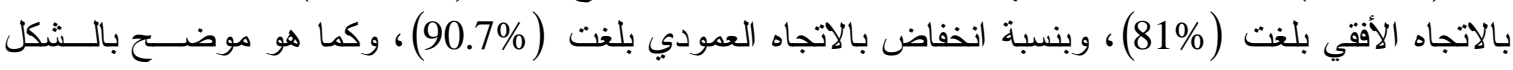

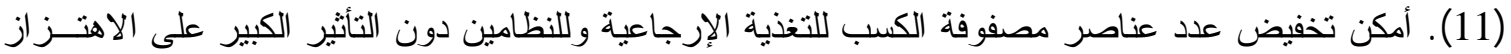

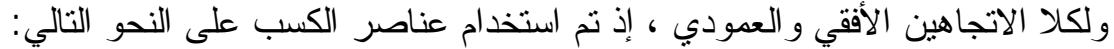

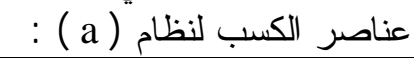

\begin{tabular}{|c|c|c|}
\hline & $x$ & $z$ \\
\hline$(K)_{\text {act }_{2}}$ & $4.3059 \times 10^{7}$ & $1.1514 \times 10^{8}$ \\
\hline
\end{tabular}

\begin{tabular}{|c|c|c|}
\hline \multicolumn{2}{|c|}{$:$ ( b ) 1 ( لناصر الكسب لنظام } \\
\hline$(K)_{a c t_{1}}$ & $\theta$ & $7.4432 \times 10^{7}$ \\
\hline
\end{tabular}

وباستخدام هذه العناصر لمصفوفتي الكسب للسيطرة على النظام الغلي ، كانت نسبة الانخفاض بالاتجــاه الأفقي (81.4\%) ونسبة الانخفاض بالاتجاه العمودي (90.1\%) ، وكما هو موضح بلى بالثكل (12). لتصبح أقطاب

$$
\begin{aligned}
& \text { النظام a } \\
& s_{5}=-15149 \quad \text { ، } \quad s_{6}=-533.69 \quad \text { ، } \quad s_{7,8}=-859.79 \mp 1172.3 i \quad \text { : b a }
\end{aligned}
$$

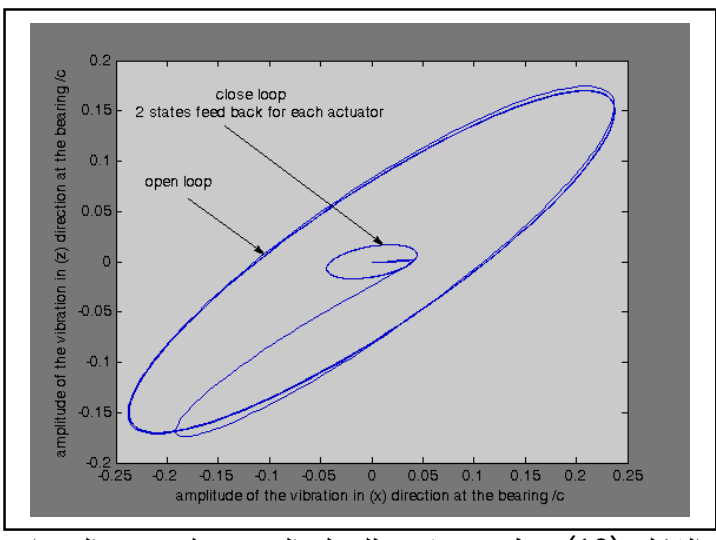

الثكل (12) منظــر جانبي للمحل الهندسي لـــعود الدوران

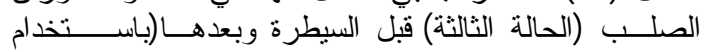

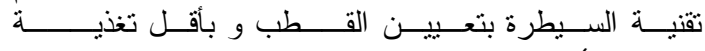

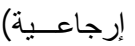

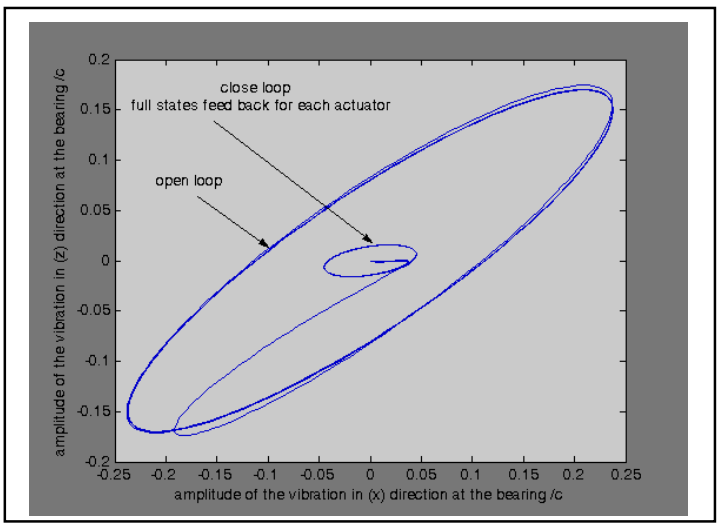

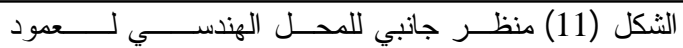

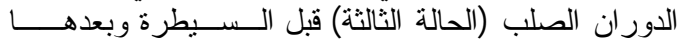

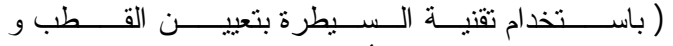

بتغذيــــــة إرجاعـية كاملة)

استخدام تقتية السيطرة المثالية :إن إيجاد عناصر المصفوفة (K) باستخدام هذه التقنية يعتمد على أساس تصغير دليل الأداء (J) إلى أقل قدر ممكن ، و المعرف بالعة العلاقة التالية [13]: $J=\int_{0}^{\infty} x(t)^{T} Q x(t)+u(t)^{T} R u(t) d t$

Q : مصفوفة الوزن لمتغير ات الحالة التي يجب أن تكون موجبة . R : مصفوفة الوزن للاخل $R(t)$ : التي يجب أن تكون موجبة أيضاً . 
إن الحد|

الطاقة المصروفة في السيطرة من خلال تقليل كلفة الداخل (t)

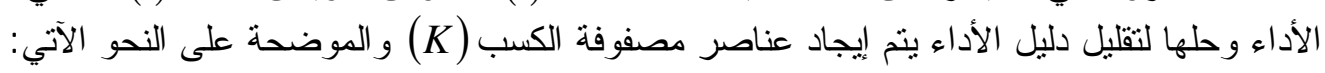

وينم استخلاص قيمة المصفوفة (P) في الحل الجبري لمعادلة ريكاتي

equation)

$A^{T} P+P A-P B R^{-1} B^{T} P+Q=0$

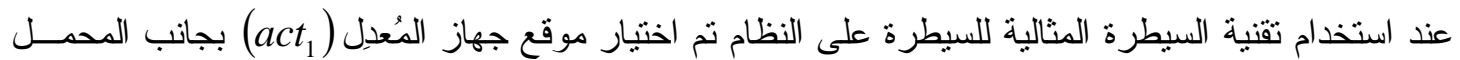

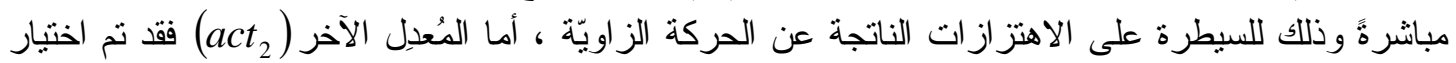

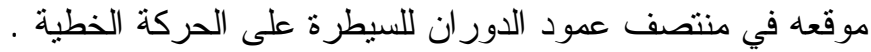
$Q=\left[\begin{array}{cccccccc}1 & 0 & 0 & 0 & 0 & 0 & 0 & 0 \\ 0 & 1 & 0 & 0 & 0 & 0 & 0 & 0 \\ 0 & 0 & 1 & 0 & 0 & 0 & 0 & 0 \\ 0 & 0 & 0 & 1 & 0 & 0 & 0 & 0 \\ 0 & 0 & 0 & 0 & 10^{5} & 0 & 0 & 0 \\ 0 & 0 & 0 & 0 & 0 & 10^{5} & 0 & 0 \\ 0 & 0 & 0 & 0 & 0 & 0 & 10^{7} & 0 \\ 0 & 0 & 0 & 0 & 0 & 0 & 0 & 10^{10}\end{array}\right]$ تم اختيار مصفوفة الوزن لمتغير ات الحالة على النحو الآتي :

وتم اختيار مصفوفة الوزن للقوى المسلطة من قِبل جهازيّ المُعدِل (act $2\left(a c t_{1}\right)$ على النحو الآتي: $R=\left[\begin{array}{cc}0.001 & 0 \\ 0 & 0.001\end{array}\right]$

تم الحصول على عناصر مصفوفتي الكسب للتغذية الإرجاعية ، وكانت هذه العناصر مع متغيرات الحالة الخاصة بها موضحة على النحو الآتي :

\begin{tabular}{|l|c|c|c|c|c|c|c|l|}
\hline & $x$ & $z$ & $\theta$ & $\varphi$ & $\dot{x}$ & $\dot{z}$ & $\dot{\theta}$ & $\dot{\varphi}$ \\
\hline$(K)_{\text {act }_{1}}$ & $4.56 \times 10^{5}$ & $1.023 \times 10^{5}$ & $1.03 \times 10^{7}$ & $-5.303 \times 10^{6}$ & $2.6 \times 0^{5}$ & $2.58 \times 10^{5}$ & $2.22 \times 10^{6}$ & $2.1 \times 10^{6}$ \\
\hline$(K)_{\text {act }_{2}}$ & $5.35 \times 10^{6}$ & $-2.65 \times 10^{6}$ & $-1.7 \times 10^{6}$ & $6.875 \times 10^{5}$ & 60372 & 34293 & 23085 & 25938 \\
\hline
\end{tabular}

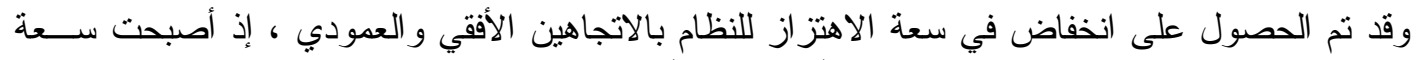
الاهتز از لعمود الدور ان بالاتجاه الأفقي تتر اوح بين (

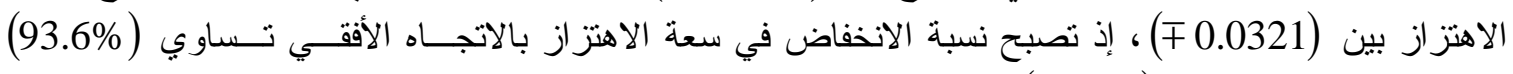

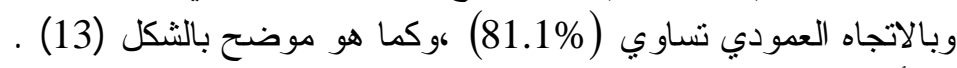

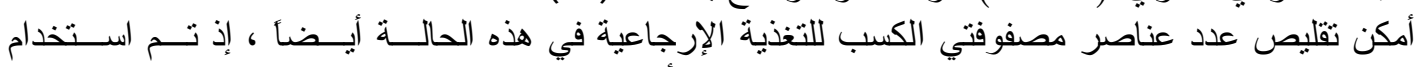
عناصر الكسب مع متغير ات الحالة الخاصة بها شريطة أن تتضمن طوري الحركتين الخطية والز اوية وكما 
محمد : السيطرة الفعالة على الاهتزازات لاوار صلا يستثد بصورة مرنة على محامل مزيتة

\begin{tabular}{|c|c|c|}
\hline & $z$ & $\varphi$ \\
\hline$(K)_{a c t_{1}}$ & $1.023 \times 10^{5}$ & $-5.303 \times 10^{6}$ \\
\hline
\end{tabular}

\begin{tabular}{|c|c|}
\hline & $x$ \\
\hline$(K)_{\text {act }_{2}}$ & $5.35 \times 10^{6}$ \\
\hline
\end{tabular}

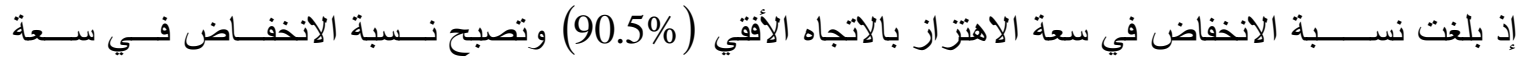

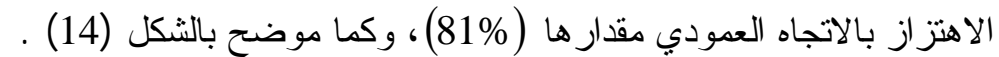

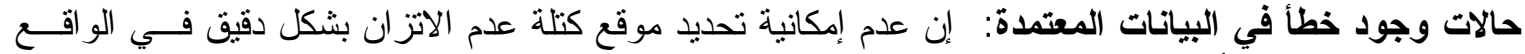

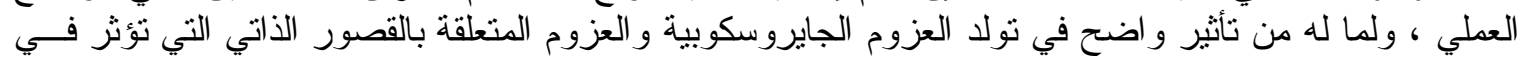

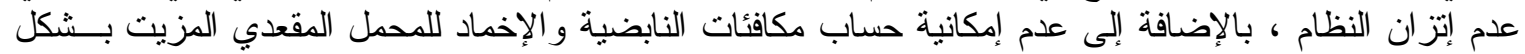

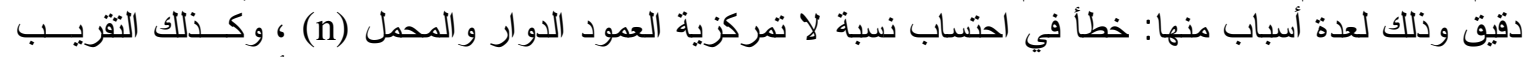

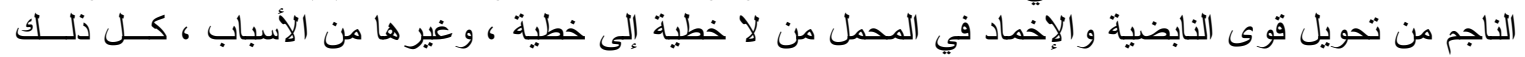

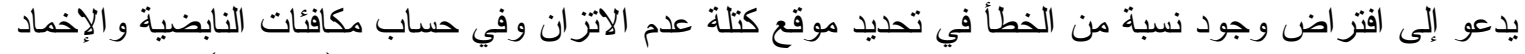

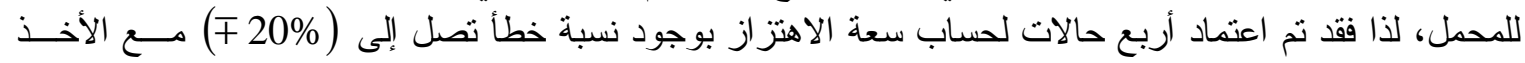

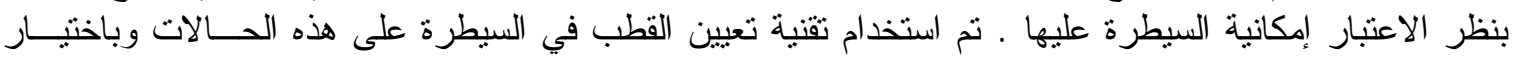

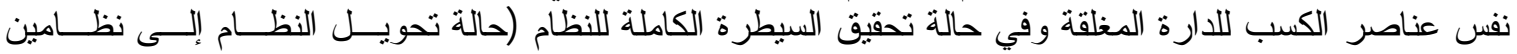

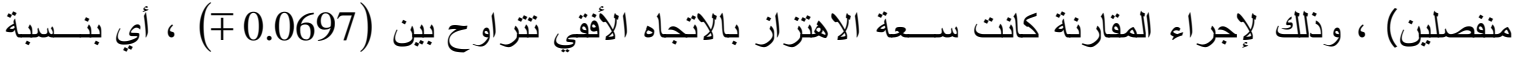

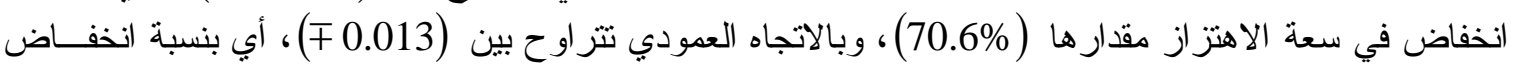

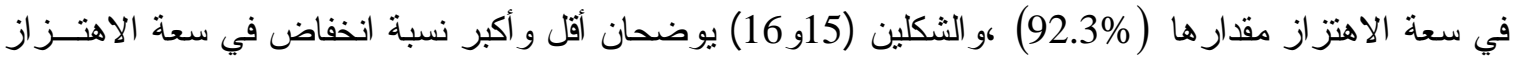

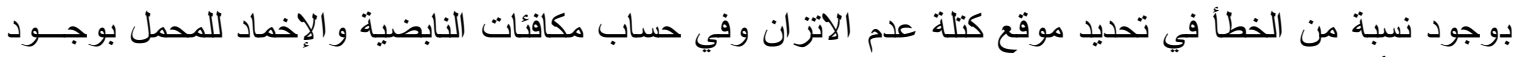

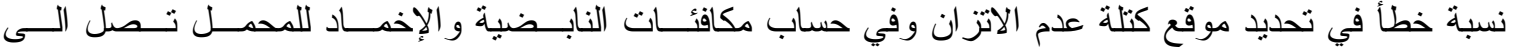

. (干 20\%)

من خلال سعة الاهتز از المســتحصلة للنظام يتبين أنه يمكن للمنظومة أن تعمل في الواقع حتى ولو كانت نســبة

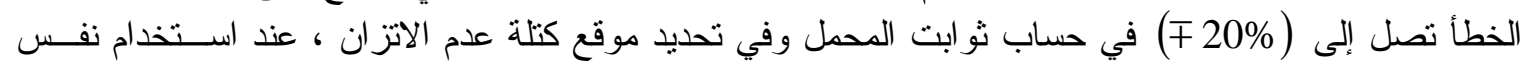

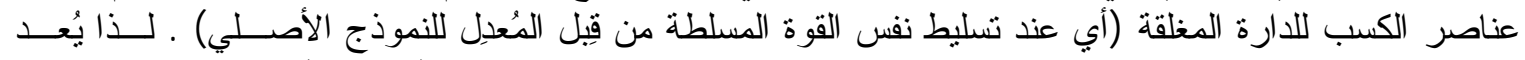
النموذج الأصلي ساري المفعول وقابلا للنطبيق العملي بوجود نسبة خطأ مقدار ها (20\% (

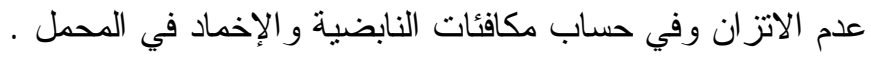

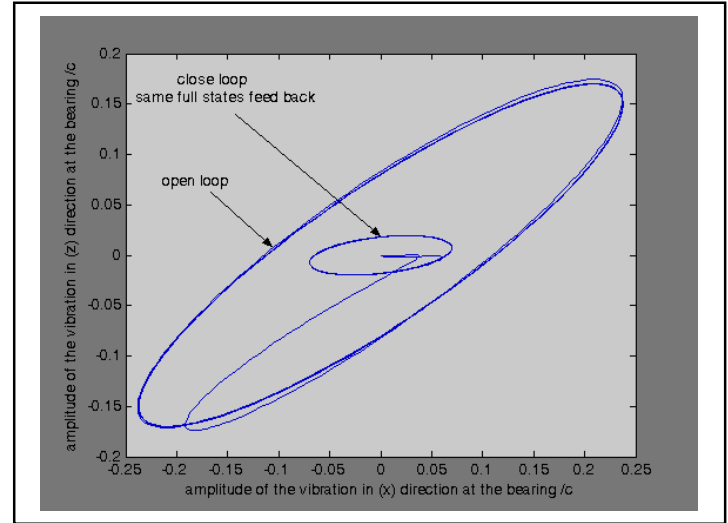

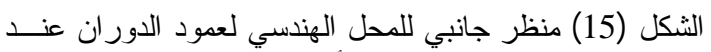

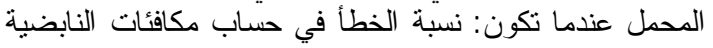

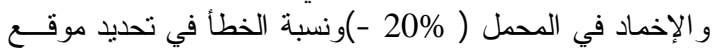
كتلة عدم الاتزان ( 20\% في الإنمل (20\%).

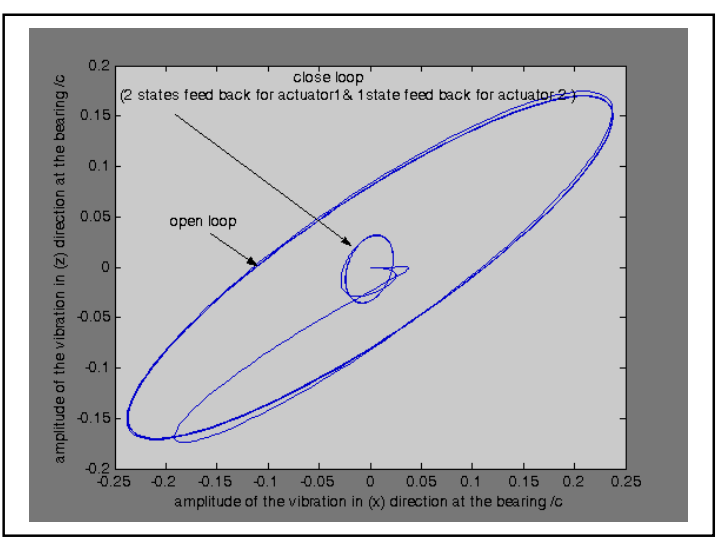

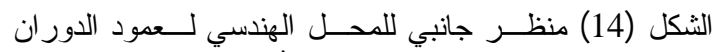

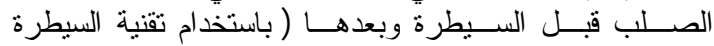

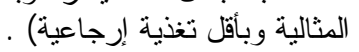




\section{$\begin{array}{llll}\text { Al-Rafidain Engineering } & \text { Vol.17 } & \text { No.5 } & \text { October } 2009\end{array}$}

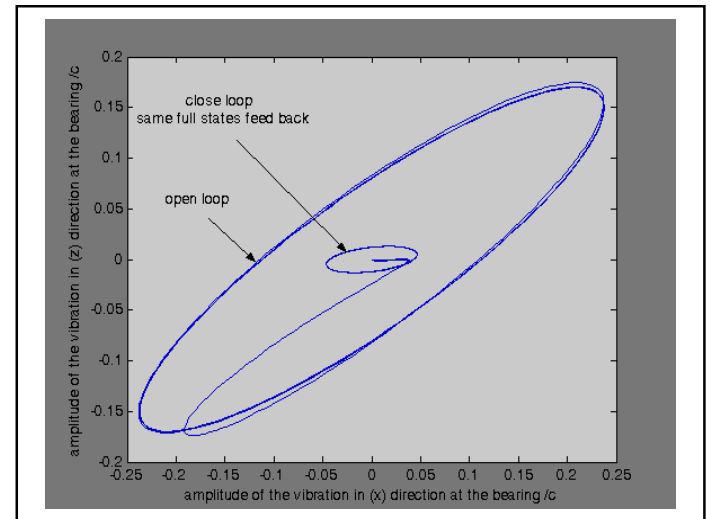

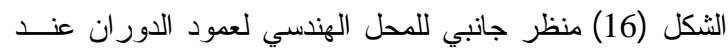

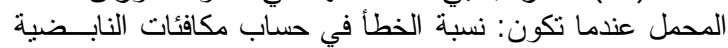
و الإخماد في المحمل ( 20\% - (20\% نسبة الخطأ في تحديد موقع كتلة عدم الاتز ان ( 20\% - (-).

(المناقشة:

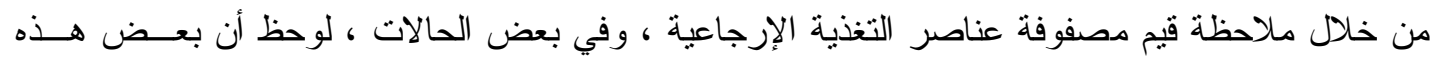

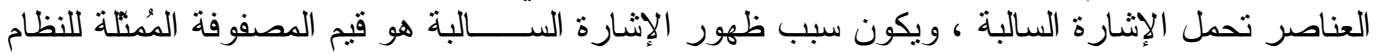

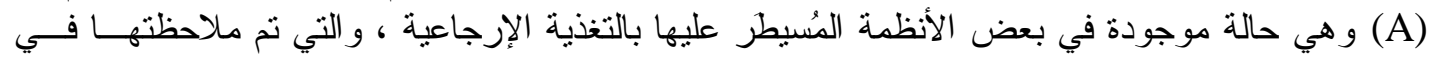

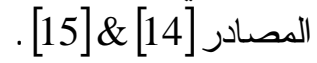

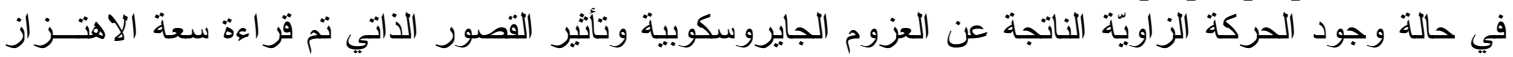

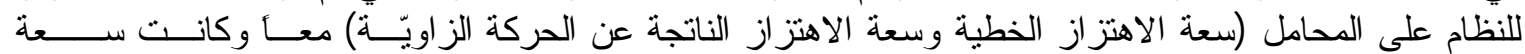

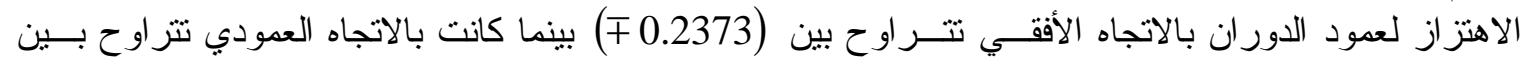

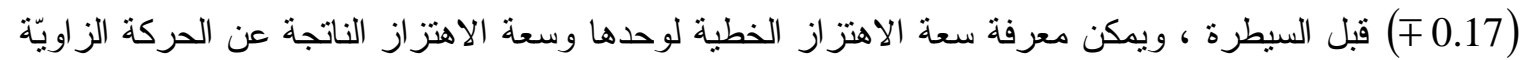

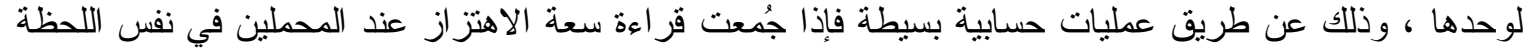

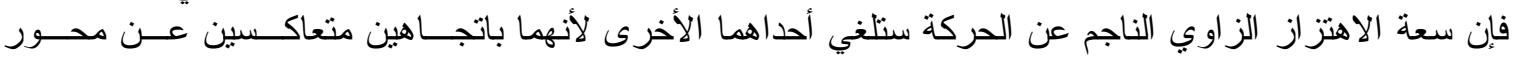

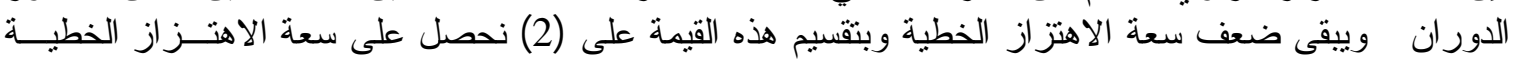

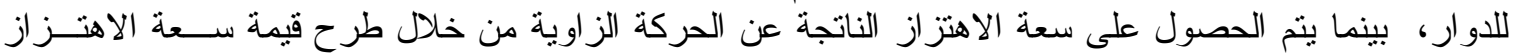

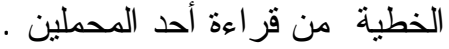

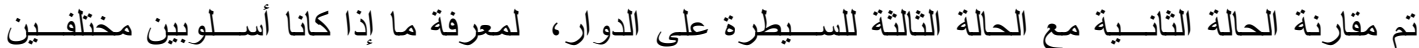

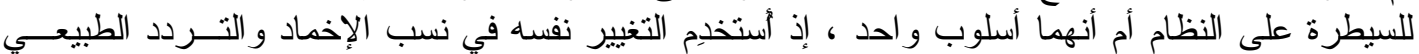

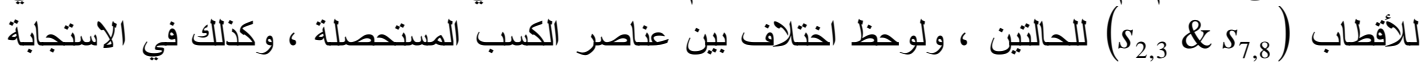

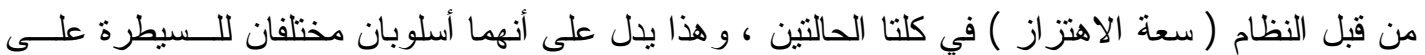
النظام.

عند النظر إلى نموذج عمود الدوران يتم ملاحظة أن العزوم الجايروسكوبية (

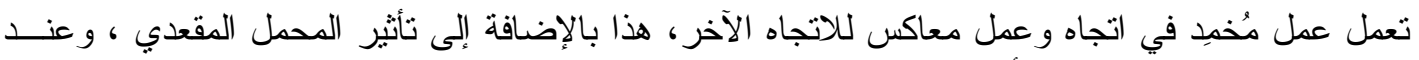

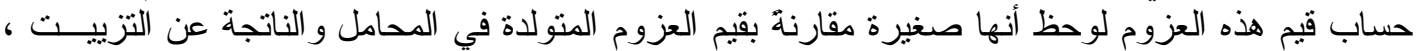
و التي تعمل على الإخماد.

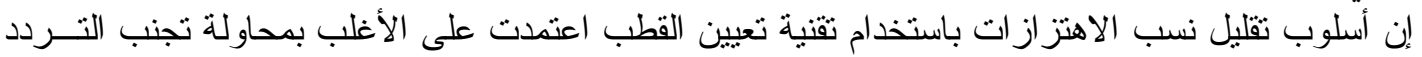

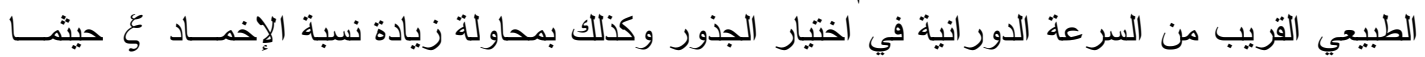

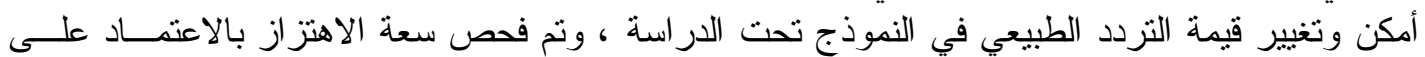

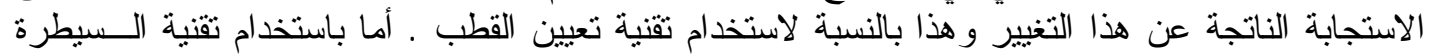

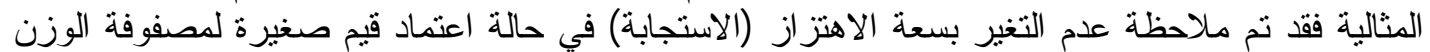

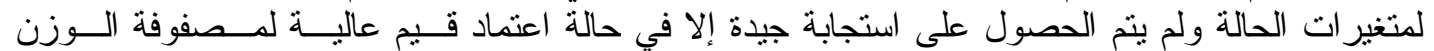

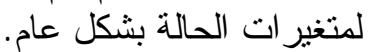




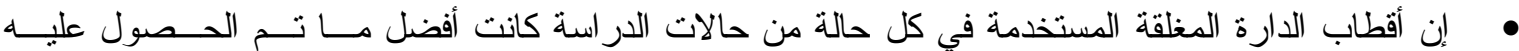
للوصول إلى عناصر مصفوفة الكسب المستخدمة في السيطرة للحصول الحئ على أفضل انخفاض في سعة الاهنز از في الاتجاهين الأفقي و العمودي.

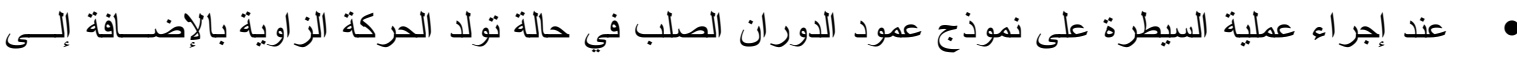

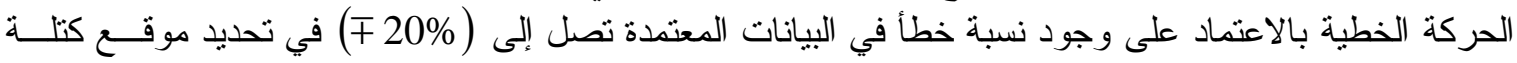

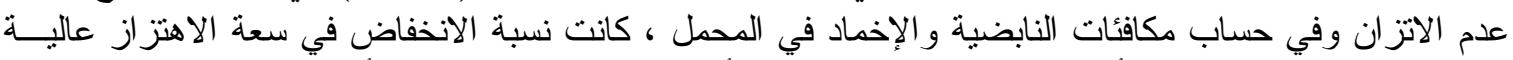

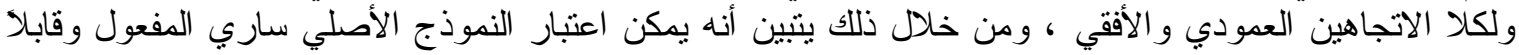
لالتطبيق العملي.

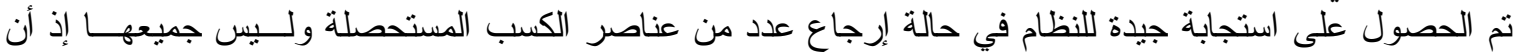

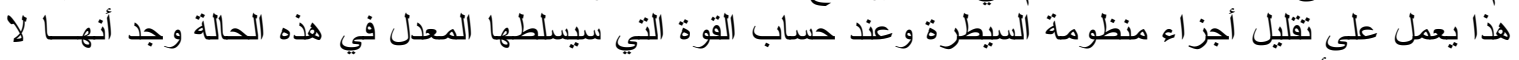

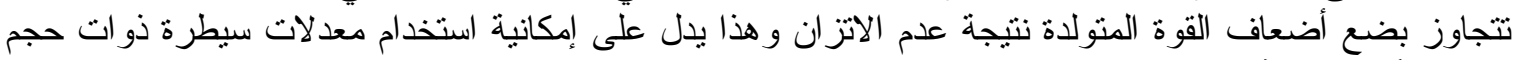
مناسب (ليس كبير أ) ، وبذلك تقل كلفة عملية السيطرة.

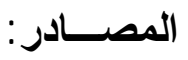

1.Stanway R. \& Jo'Reilly, "State - Variable Feed Back Control of Rotor - Bearing Suspension System", Conference of Vibration in Rotating Machinery, England, 1984.

2.Krodkiewski J. M., Sun L. \& Cen Y., " Control Law Synthesis for Self - Tuning Adaptive Control of Forced Vibration in Rotor Systems", Proc. of Second International Symposium MV2 on Active Control in Mechanical Engineering, Vol. 1, PP: S9-25-37, Lyon, France, Oct. 22-23, 1997.

3.Krodkiewski J. M., Sun L. \& Cen Y., "Improvement of Stability of Rotor System by Introducing a Hydraulic Damper into An Active Journal Bearing”, International Journal of Rotating Machinery, Vol. 3 No. 1, PP: 45-52,England, 1997.

4.Zhou S. \& Shi J., "Supervisory Adaptive Balancing of Rigid Rotors During Acceleration", Transactions of NAMRI / SME, Vol. 27, PP: 425 - 430, 2000.

5.Zhou S. \& Shi J., "Optimal One - Plane Active Balancing of Rigid Rotor During Acceleration", Journal of Sound and Vibration, 2001.

6.Zhou S. \& Shi J., "Imbalance Estimation for Speed - Varying Rigid Rotors Using Time Varying Observer", ASME Transactions Journal of Dynamic Systems, Measurements and Control, U.S.A., 2002.

7. Zhou S., Shin K., Dyer S.W., Shi J. \& Ni J., "Extended Influence Coefficient Method for Rotor Active Balancing During Acceleration", The Shock and Vibration Digest, Vol. 33, No. 4, U.S.A., 2001.

8.Yamamoto T. \& Ishida Y., "Linear and non Linear Rotor Dynamics", John Wiley \& Sons, Inc., New York, U.S.A, 2001.

9. Dimarogonas A., “Vibration for Engineers", Prentice-Hall, International, Inc., New Jersey, U.S.A, 1996.

10.Pandya N. C. \& Shah C. S., “Elements of Machine Design”, Charotar Publishing House, India, 1986.

11.Basheer I. A., “ Active Control of Vibration in Rotor-Bearing System ”,MSc. Thesis, University of Mosul -college of Engineering, Mechanical Engineering Dept., Iraq, 2005.

12.Helio F. de Castro, et al.," Experimental Performance Evaluation of Magnetic Actuator used in Rotating Machinery Analysis", J. of Braz. ,Soc. of Mech. Sci and Eng., Vol. 29, No. 1/101, Jan-March, Brazil, 2007. 


\section{$\begin{array}{llll}\text { Al-Rafidain Engineering } & \text { Vol.17 } & \text { No.5 } & \text { October } 2009\end{array}$}

13. Kwakernaak H. \& Sivan R., "Linear Optimal Control Systems", John Wiley \& Sons, Inc., U.S.A, 1972.

14. Borrie J. A., “ Modern Control Systems ", Prentice-Hall, International, Inc., London, U.K, 1986.

15. Franklin G. F., Powell J. D. \& Workman M. “ Digital Control of Dynamic Systems”, Addison- Wesley, Inc., England, 1998. 